Frank Schweitzer, Laxmidhar Behera, Heinz Mühlenbein:

Evolution of Cooperation in a Spatial Prisoner's Dilemma

Advances in Complex Systems, vol. 5, no. 2-3, pp. 269-299

\title{
Evolution of Cooperation in a Spatial Prisoner's Dilemma
}

\author{
Frank Schweitzer ${ }^{1,2}$, Laxmidhar Behera ${ }^{1,3}$, Heinz Mühlenbein ${ }^{1}$ \\ 1 Fraunhofer Institute for Autonomous Intelligent Systems, \\ Schloss Birlinghoven, 53754 Sankt Augustin, Germany \\ 2 Institute of Physics, Humboldt University, Invalidenstraße 110, \\ 10115 Berlin, Germany, schweitzer@physik.hu-berlin.de \\ 3 Department of Electrical Engineering, Indian Institute of Technology \\ Kanpur 208 016, India, Ibehera@iitk.ac.in
}

\begin{abstract}
We investigate the spatial distribution and the global frequency of agents who can either cooperate or defect. The agent interaction is described by a deterministic, non-iterated prisoner's dilemma game, further each agent only locally interacts with his neighbors. Based on a detailed analysis of the local payoff structures we derive critical conditions for the invasion or the spatial coexistence of cooperators and defectors. These results are concluded in a phase diagram that allows to identify five regimes, each characterized by a distinct spatiotemporal dynamics and a corresponding final spatial structure. In addition to the complete invasion of defectors, we find coexistence regimes with either a majority of cooperators in large spatial domains, or a minority of cooperators organized in small non-stationary domains or in small clusters. The analysis further allowed a verification of computer simulation results by Nowak and May (1993). Eventually, we present simulation results of a true 5 -person game on a lattice. This modification leads to non-uniform spatial interactions that may even enhance the effect of cooperation.

Keywords: Prisoner's dilemma; cooperation; spatial 5-person game
\end{abstract}

\section{Introduction}

The evolution of cooperation has been extensively studied in a biological, social and ecological context [2, 1, 9, 10, 11, 15, 16, 18, 25, 36, 40, 41, 44]. In order to obtain a general theory for this, in particular the so called Prisoner's Dilemma (PD) (see also Sect. 2.2) - an evolutionary game introduced by Rapoport and Chammah [37] (see also [38]) - has been widely investigated [17, 8, 13, 14, 17, 20, 22, 26, 28, 30, 34, 43. Based on PD investigations, in his seminal work Axelrod 
Frank Schweitzer, Laxmidhar Behera, Heinz Mühlenbein:

Evolution of Cooperation in a Spatial Prisoner's Dilemma

Advances in Complex Systems, vol. 5, no. 2-3, pp. 269-299

[3] has shown that cooperation can emerge as a norm in a society comprised of individuals with selfish motives - for a review of this development in the last twenty years see e.g. [19].

The Prisoner's Dilemma is based on the precondition that it pays off to be not cooperative, i.e. to defect in a cooperative environment, this way taking a "free ride" at the costs of those how are cooperating, e.g. for a commong good. This type of problem has been also recognized as the "tragedy of the commons" 18]. Many investigations of the Prisoner's Dilemma consider a so-called iterated game 22, 6, 9, 14, 21, 22, 27, 35, where the players interact consecutively a given number of times, $n_{g} \geq 2$. It makes sense only if the players can remember the previous choices of their opponents, i.e. if they have a memory of $n_{m} \leq n_{g}-1$ steps. Then, they may be able to develop different strategies - such as the famous "tit for tat" [3, 5] - based on their past experiences with their opponents.

In this paper, we are only interested in a non-iterated PD game, $n_{g}=1$, which is also called a "one-shot" game. In this case the players - or the agents as we call them here - do not develop strategies, they can rather choose between two different actions, to cooperate (C) and to defect (D). It can be shown (see also Sect. 2.2) that in the one-shot game defection is the only evolutionary stable strategy (ESS) [42] if each player interacts with any other player. Such a population is called panmictic, and their dynamics can be predicted within a mean-field analysis. Interestingly, this picture changes if a spatial structure of the population is considered, i.e. if the interaction between agents is locally restricted to their neighbors [17, 20, 24, 31, 32, 35, 45]. Then, a stable spatial coexistence between cooperators and defectors becomes possible under certain conditions 32, 33, 35.

The current paper focuses on the spatial interaction of cooperators and defectors both analytically and by means of computer simulations. We consider an agent population placed on a square lattice and simulate the dynamics of the population by means of a cellular automata defined in Sect. 2.1. The interaction between the agents is modeled as a n-person game, i.e. $n$ agents interact simultaneously. In this paper, a 5-person game is considered defined by the spatial structure of each agent's four nearest neighbors. It is already known that multi-person games produce a rather complex collective dynamics [1, 11] which becomes even more difficult in the spatial case. Therefore, in Sects. 3 and 4, we derive analytically critical conditions for the invasion or the coexistence of cooperating and defecting agents. In particular, we verify analytically the critical parameters found by Nowak and May [32] by means of computer simulations. A very detailed investigation leads us to a phase diagram that allows to distinguish between five different dynamic regimes for the spatial evolution. Examples for these are demonstrated in Sect. 4 by means of computer simulations. In Sect. 5 we present a modification of the model, that to our knowledge for the first time investigates a true 5-person game on the lattice. The results indicate that these modifications may lead to an increase of cooperation in the spatial population. 


\section{Model of Cooperation}

\subsection{Defining the cellular automaton}

We consider a model of two types of agents, cooperators $(C)$ and defectors $(D)$. The disctinction is made by means of a variable $\theta \in\{0,1\}$, where 1 refers to cooperation and 0 refers to defection. Whether an individual agent $i$ belongs to the subpopulation of $C$ or $D$ is indicated by a variable, $\theta_{i} \in \theta$. The total number of agents is constant, thus the global frequency $f_{\theta}$ is defined as:

$$
\begin{aligned}
& N=\sum_{\theta} N_{\theta}=N_{0}+N_{1}=\text { const. } \\
& f_{\theta}=\frac{N_{\theta}}{N} ; f \equiv f_{1}=1-f_{0}
\end{aligned}
$$

In the following, the variable $f$ shall refer to the global frequency of the cooperating agents.

For the spatial distribution of the agents let us consider a two-dimensional cellular automaton (CA) (or a two-dimensional square lattice) consisting of $N$ cells. Since the cells can be numbered consecutively, each cell is identified by the index $i \in N$, that also refers to its spatial position. We note that in most two-dimensional CA the position of the cells are identified by $i j$ coordinates referring to the two dimensions, however in order to define neighborhoods and to simplify the notations, we will use only index $i$ for the spatial position. This implies that the description presented below may also apply to one-dimensional CA provided the neighborhoods are defined accordingly.

Each cell shall be occupied by one agent, thus each cell is characterized by a discrete value $\theta_{i} \in\{0,1\}$ indicating whether it is occupied by a cooperator or a defector. The spatiotemporal distribution of the agents is then described by

$$
\Theta=\left\{\theta_{1}, \theta_{2}, \ldots, \theta_{N}\right\}
$$

Note that the state space $\Omega$ of all possible configurations is of the order $2^{N}$.

For the spatial evolution of $\Theta$ described in the following, we have to consider the occupation distribution of the local neighborhood that surrounds each cell $i$. Let us define the size of a neighborhood by $n$ (that also includes cell $i$ ), then the different neighbors of $i$ are characterized by a second index $j=1, \ldots, n-1$, where the numbering starts with the nearest neighbors (cf. Fig. 1). Note that $\theta_{i} \equiv \theta_{i_{0}}$ (i.e $j=0$ ). The number of nearest neighbors of cell $i$ shall be denoted as $m$, whereas the number of only the second nearest neighbors is denoted as $r$. Obviously, $n=m+r+1$. For further use, we define the local occupation $\underline{\theta}_{i}$ of the nearest neighborhood (without cell $i$ ) as:

$$
\underline{\theta}_{i}=\left\{\theta_{i_{1}}, \theta_{i_{2}}, \ldots, \theta_{i_{m}}\right\}
$$


Figure 1: Sketch of the two-dimensional cellular automaton. It shows a neighborhood of size $n$ of cell $i$ where the neighbors are labeled by a second index $j=1, \ldots, n-1$. The value $\theta_{i_{j}} \in\{0,1\}$ indicates whether the cell is occupied either by a defector or a cooperator. The $m$ nearest neighbors are shown in darker gray, the $r$ second nearest neighbors in lighter gray.

\subsection{Rules of the Game}

In order to specify the interaction between the agents, we adopt a well known prototype from game theory. It assumes that each agent $i$ has two options to act in a given situation, to cooperate $(C)$, $\theta_{i}=1$, or to defect $(D), \theta_{i}=0$. Playing with agent $j$, the outcome of this interaction depends on the action chosen by agent $i$, i.e. $C$ or $D$, without knowing the action chosen by the other agent participating in a particular game. It is described by a payoff matrix, which for the 2-person game has the following form:

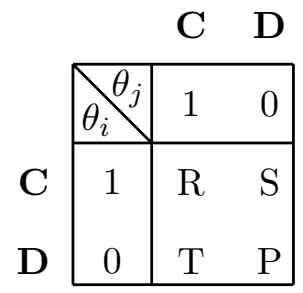

Suppose, agent $i$ has chosen to cooperate, then its payoff is $R$ if the other agent $j$ has also chosen to cooperate (without knowing about the decision of agent $i$ ), but $S$ if agent $j$ defects. On the other hand, if agent $i$ has chosen not to cooperate, then it will receive the payoff $T$ if agent $j$ cooperates, but $P$ if agent $j$ defects, too. 
Frank Schweitzer, Laxmidhar Behera, Heinz Mühlenbein:

Evolution of Cooperation in a Spatial Prisoner's Dilemma

Advances in Complex Systems, vol. 5, no. 2-3, pp. 269-299

In a special class of games, the so-called Prisoner's Dilemma (PD), the payoffs have to fulfill the following two inequalities:

$$
\begin{array}{r}
T>R>P>S \\
2 R>S+T
\end{array}
$$

The known standard values are $T=5, R=3, P=1, S=0$. This means in a cooperating environment, a defector will get the highest payoff. From this, the abbreviations for the different payoffs become clear: $T$ means (T)emptation payoff for defecting in a cooperative environment, $S$ means (S)ucker' payoff for cooperating in a defecting environment, $R$ means (R)eward payoff for cooperating in a likewise environment, and $P$ means $(\mathrm{P})$ unishment payoff for defecting in a likewise environment.

In any one round (or "one-shot") game, choosing action $D$ is unbeatable, because it rewards the higher payoff for agent $i$ whether the opponent chooses $C$ or $D$. At the same time, the payoff for both agents $i$ and $j$ is maximized when both cooperate.

A simple analysis shows that defection is a so-called evolutionary stable strategy (ESS) in a oneshot PD. If any two agents are able to interact and the number of cooperators and defectors in the population is given by $N_{1}$ and $N_{0}=N-N_{1}$ respectively, then the expected average payoff for cooperators will be $\bar{a}_{1}=\left(R \times N_{1}+S \times N_{0}\right) / N$. Similarly the expected average payoff for defectors will be $\bar{a}_{0}=\left(T \times N_{1}+P \times N_{0}\right) / N$. Since $T>R$ and $P>S, \bar{a}_{0}$ is always larger than $\bar{a}_{1}$ for a given number $N_{1}$, and pure defection would be optimal in a one-shot game. Even one defector is sufficient to invade the complete population of $N-1$ cooperators.

This conclusion holds for a so called panmictic population where each agent interacts with all other $N-1$ agents. But in this paper, we are mainly interested in the spatial effects of the PD game. Therefore, let us assume that each agent $i$ interacts only with the $m$ agents of its neighborhood, eq. (3). In evolutionary game theory this is called a $n$-person game, where $n=m+1=5$ in the given case. Each game is played between $n$ players simultaneously, and the payoff for each player depends on the number of cooperators in its group, $s \leq n$. Then the payoff matrix, eq. (四) has to be extended according to the number of players, but it still has to fullfill the following conditions according to the Prisoner's Dilemma rationale 46:

1. Given a fixed number $s$ of cooperators in the group, defection pays always more than cooperation:

$$
a_{0}^{s}>a_{1}^{s} ; \quad s=0,1,2, \ldots, n-1
$$

where $a_{0}^{s}$ is the payoff for each defector in the group of size $n$ and $a_{1}^{s}$ is the payoff for each cooperator.

2. The payoff increases for both cooperators and defectors, as number $s$ of cooperators in the group increases:

$$
a_{1}^{s}>a_{1}^{s-1} ; \quad a_{0}^{s}>a_{0}^{s-1} ; \quad s=1,2, \ldots, n-1
$$


Frank Schweitzer, Laxmidhar Behera, Heinz Mühlenbein:

Evolution of Cooperation in a Spatial Prisoner's Dilemma

Advances in Complex Systems, vol. 5, no. 2-3, pp. 269-299

3. The average payoff of the group increases with an increasing number of cooperators:

$$
a_{1}^{s} s+a_{0}^{s}(n-s)>a_{1}^{s-1}(s-1)+a_{0}^{s-1}(n-s+1)
$$

There are various ways to fulfill the conditions (7)-(9). One simple method uses the concept of the 2-person game described above to calculate the payoff of an agent in the $n$-person game from the accumulated payoffs of $(n-1) 2$-person games. I.e. for a neighborhood of 5, the 5-person game is decomposed into 2-person games, that may occur independently, but simultaneously [23]. This method will be also used in the current paper.

With respect to the spatial interaction in the neighborhood, we will further assume that each agent interacts only with the $m$ nearest neighbors [32]. In Sect. 5, we will also shortly discuss the case where each agent in the neighborhood of size $n$ interacts with every other agent in this neighborhood (which sometimes also involves interaction with second nearest neighbors, as Fig. 1 indicates). These assuptions will allow us to further use the payoff matrix of the 2-person game, eq. (4), and to reduce the interaction in the neighborhood of size $n$ to $n-1$ simultaneous 2-person games played by agent $i$.

In order to introduce a time scale, we define a generation $G$ to be the time in which each agent has interacted with its $m$ nearest neighbors a given number of times, denotes as $n_{g}$. In our case $n_{g}=1$ (which is called a one-shot game), thus the total number of interaction during each generation is roughly $N m / 2$. We note here that $n_{g}>1$ can play an important role for the invasion of cooperation into a heterogeneous population, [6, 14].

We assume that during each generation the state $\theta_{i}$ of an agent is not changed while it interacts with its neighbors simultaneously. But after a generation is completed, $\theta_{i}$ can be changed based on the following considerations: From the interaction with its neighbors, each agent $i$ receives a payoff $a_{i}$ that depends both on its current state $\theta_{i}$, i.e. whether agent $i$ has cooperated or not, and on the $\theta_{i_{j}}$ of its neighbors. The fraction of cooperators and defectors in the neighborhood of agent $i$ is given by:

$$
z_{i}^{\theta}=\frac{1}{m} \sum_{j=1}^{m} \delta_{\theta \theta_{i_{j}}} ; \quad z_{i}^{(1-\theta)}=1-z_{i}^{\theta} \quad(\theta \in\{0,1\})
$$

where $\delta_{x y}$ means the Kronecker delta, which is 1 only for $x=y$ and zero otherwise. According to the payoff matrix of eq. (4), the payoff of agent $i$ is then defined as:

$$
a_{i}\left(\theta_{i}\right)=\delta_{1 \theta_{i}}\left[z_{i}^{1} R+z_{i}^{0} S\right]+\delta_{0 \theta_{i}}\left[z_{i}^{1} T+z_{i}^{0} P\right]
$$

Note that the additive calculation of the payoff according to eqs. (10), (11) is based on the assumtion of the $m$ simultaneous, but independent 2-person games played by agent $i$.

The payoff $a_{i}$ is then compared to the payoffs $a_{i_{j}}$ of all neighboring agents, in order to find the maximum payoff within the local neighborhood during that generation, $\max \left\{a_{i}, a_{i_{j}}\right\}$. If agent $i$ 
Frank Schweitzer, Laxmidhar Behera, Heinz Mühlenbein:

Evolution of Cooperation in a Spatial Prisoner's Dilemma

Advances in Complex Systems, vol. 5, no. 2-3, pp. 269-299

has received the highest payoff, then it will keep its $\theta_{i}$, i.e. it will continue to either cooperate or to defect. But if one of its neighbors $j$ has received the higher payoff, then agent $i$ will adopt the behavior of the respective agent. This also implies that it may keep its previous behavior if the more successful agent has the same $\theta_{j}$. If

$$
j^{\star}=\arg \max _{j=0, \ldots, m} a_{i_{j}}
$$

defines the position of the agent that received the highest payoff in the neighborhood, the update rule of the game can be concluded as follows:

$$
\theta_{i}(G+1)=\theta_{i_{j^{\star}}}(G)
$$

which means specifically:

$$
\theta_{i}(G+1)=\left\{\begin{array}{cl}
\theta_{i}(G) & \text { if } \theta_{i}(G)=\theta_{i_{j^{\star}}}(G) \\
1-\theta_{i}(G) & \text { otherwise }
\end{array}\right.
$$

We note that the evolution of the system described by eq. (14) is completely deterministic, results for stochastic CA have been discussed in [12, 29, 39].

\subsection{First insights from the CA simulations}

Eventually, to give an impression of the spatial dynamics that arises from the game desribed above, we have conducted a computer simulation of the CA, using periodic boundary conditions and a simultaneous update. The global frequency of cooperators was initially set to $f(0)=0.5$. The results are shown in the time series of Fig. 2 and the time dependence of $f(G)$ in Fig. 3 .

From the computer simulations, we notice the following interesting features of the spatial game: Starting with an initial random distribution of cooperators and defectors, we observe the formation of spatial domains dominated by either cooperators or defectors. In the very early stages of evolution, the cooperators concentrate in only a few small clusters that are like islands in the sea of defectors. But then these cooperating clusters increase in size, i.e. the cooperators invade into the domains of defectors, until in the long run they are the global majority. At all times, we observe a spatial coexistence between cooperators and defectors in different domains, instead of a complete spatial separation into two domains. We further notice that in the long run the spatial pattern becomes stationary (with very small periodic changes that can be also noticed in Fig. 3).

In order to generalize the above results that have been obtained from only a single run of the CA, we need to answer the following questions:

- Under what conditions will there be a spatial coexistence of cooperators and defectors, and when will we observe the extinction of either cooperators or defectors? 

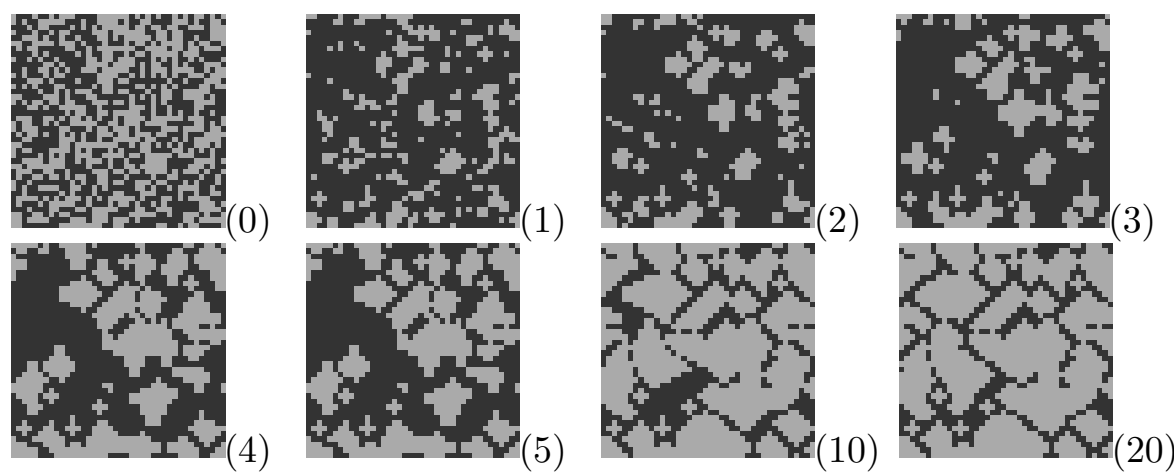

Figure 2: Time series of the spatial distribution of cooperators (grey) and defectors (black) on a CA of size $N=40 \times 40$. The time is given by the numbers of generations in brackets. Initial condition: $f(0)=0.5$, random spatial distribution of cooperators and defectors. Parameters for the payoff matrix, eq. (田): $\{R ; S ; T ; P\}=\{3.0 ; 0.0 ; 3.5 ; 0.5\}$ (region A, see Sect. 4.1).

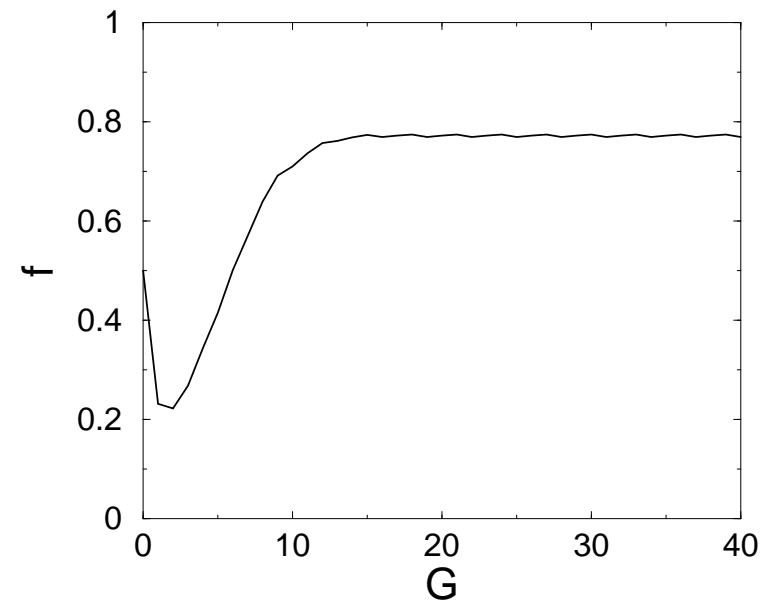

Figure 3: Global frequency of cooperators, $f$, eq. (1), vs. time (number of generations). The data have been obtained from the time series of Fig. 2 .

- What does the variety and the size of the spatial domains depend on?

- Will the dynamics always lead to stationary patterns, or will there be also non-stationary patterns in the long run?

- How does the dynamics changes if a larger local neighborhood is considered in the update rule (i.e. if the second nearest neighbors are included, too)?

These questions will be investigated in detail in the following sections. In particular, we will derive 
Frank Schweitzer, Laxmidhar Behera, Heinz Mühlenbein:

Evolution of Cooperation in a Spatial Prisoner's Dilemma

Advances in Complex Systems, vol. 5, no. 2-3, pp. 269-299

analytical results for the conditions that need to be fulfilled by the payoff matrix, eq. (四) in order to find certain spatial patterns.

\section{Invasion vs. coexistence of cooperating and defecting agents}

\subsection{Payoffs for local configurations}

As pointed out in the previous section, the change of agent $i$ 's "behavior", $\theta_{i} \rightarrow\left(1-\theta_{i}\right)$, depends on the payoff of the agents in the immediate neighborhood, which in turn depends on the local occupation, $\underline{\theta}_{i}$, eq. (3). In order to derive analytical conditions for the transition of $\theta_{i}$, we thus have to look more closely at the possible local occupation patters that shall in the following be described by a term $K_{\theta}^{s}$. Here, $\theta \in\{0,1\}$ describes whether the center cell is occupied by either a defector or a cooperator, and $s \in\{0,1,2,3,4\}$ gives the total number of cooperators in the nearest neighborhood. Examples for possible configurations in the neighborhood of a defector are shown in eq. (15):

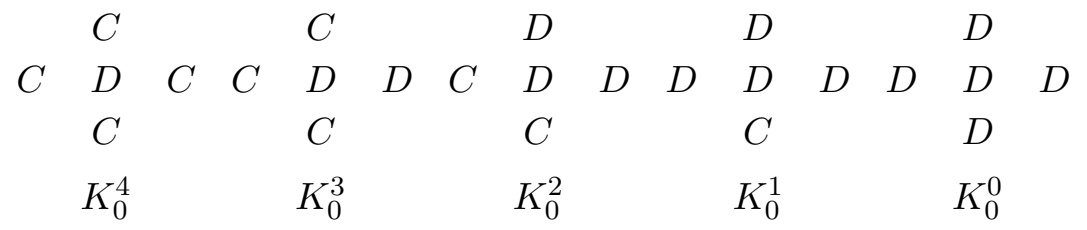

Of course, for any given number $s \in\{1,2,3\}$, there are different local occupation patterns possible that may result from exchanging the positions of the agents in the neighborhood. But, as we have seen from eq. (11), it is not really the position of agents that matters here, but only the number of cooperators and defectors in the local neighborhood, eq. (10). Therefore, $K_{0}^{s}$ provides sufficient information about the local configuration. The possible configurations for $K_{1}^{s}$, where a cooperator occupies the center cell are analogous to eq. (15).

For each possible realization of $K_{\theta}^{s}$, the respective payoff of the agent in the center, $a_{\theta}^{s}$ can be calculated according to eq. (11). The results are given by eq. (16) for cooperators and defectors both in terms of an analytical expression and for a particular realization of the payoff matrix, eq. (4), $\{R ; S ; T ; P\}=\{3 ; 0 ; 5 ; 1\}$ (which are the "classical" values):

$$
\begin{array}{llllll}
\multicolumn{3}{c}{\text { cooperator }} & & \text { defector } & \\
a_{1}^{4}= & R & =3.0 & a_{0}^{4}= & T & =5.0 \\
a_{1}^{3}=\frac{3 R+S}{4} & =2.25 & a_{0}^{3}= & \frac{3 T+P}{4} & =4.0 \\
a_{1}^{2}=\frac{2 R+2 S}{4} & =1.5 & a_{0}^{2}= & \frac{2 T+2 P}{4} & =3.0 \\
a_{1}^{1}=\frac{R+3 S}{4} & =0.75 & a_{0}^{1}= & \frac{T+3 P}{4} & =2.0 \\
a_{1}^{0}= & S & =0.0 & a_{0}^{0}= & P & =1.0
\end{array}
$$


Frank Schweitzer, Laxmidhar Behera, Heinz Mühlenbein:

Evolution of Cooperation in a Spatial Prisoner's Dilemma

Advances in Complex Systems, vol. 5, no. 2-3, pp. 269-299

From eq. (16), we notice that the payoff $a_{\theta}^{s}$ increases with the number of cooperators $s$ in the neighborhood, i.e. for a particular $\theta$

$$
a_{\theta}^{s}>a_{\theta}^{s-1} \quad(s \in\{1, \ldots, 4\})
$$

yields. Further, taking the relations of eqs. (5), (6) into account, it is obvious that always

$$
\begin{aligned}
& a_{0}^{4}=\max \left\{a_{0}^{s}, a_{1}^{s}\right\} \\
& a_{1}^{0}=\min \left\{a_{0}^{s}, a_{1}^{s}\right\} \\
& a_{0}^{s}>a_{1}^{s} \quad(s \in\{0,1, \ldots, 4\})
\end{aligned}
$$

Comparing eqs. (17), (18) with the conditions (7)-(9), we can also verify that the payoffs in the current form fulfill the general requiremements for the n-person PD. Based on these general considerations, in the following we discuss the different conditions that arise in the spatial case for the invasion or the coexistence of cooperators and defectors.

\subsection{Conditions for invasion and coexistence}

Basically, the update rule of eq. (14) determines whether a cell is "invaded" by a particular subpopulation (i.e. whether the agent adopts the respective behavior). It depends on the local payoff received in comparison with the payoffs of the neighboring sites. In order to find the conditions for invasion and coexistence, we need to concentrate on the border region between the domains of

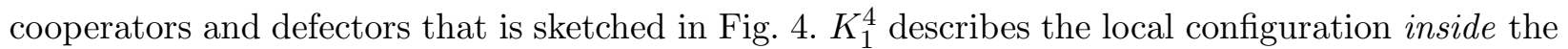
domain of cooperators, while $K_{0}^{0}$ describes the local configuration inside the domain of defectors. Further, the possible configurations that can be found only in the border region are given. The local configurations $K_{1}^{1}$ and $K_{1}^{0}$ are not listed there because they are completely unstable. These configurations receive the lowest payoff and therefore, if they initially exist, vanish within the next generation and do not need to be taken into account for the discussion of the further evolution.

In order to elucidate the dynamics at the border, we will separately discuss the two possible cases: (i) invasion of configurations $K_{0}^{s}$ "owned" by defectors into neighboring configurations $K_{1}^{s}$ "owned" by cooperators, and (ii) the reverse case. Note that the cooperator (1) and the defector (0) are always on adjacent sites. The invasion process, i.e. the occupation of the center cell by an agent of the opposite subpopulation shall be indicated by an arrow $\Rightarrow$. The results for case (i) are listed in Table 11 and are explained below.

The defective behavior can invade the whole spatial population without any exception (in a deterministic case), if the lowest possible payoff for defectors, $a_{0}^{1}$, is larger than the highest possible payoff for collaborators, $a_{1}^{4}$. Actually, the payoff $a_{0}^{0}$ does not matter here, because in the respective configuration $K_{0}^{0}$, there is no cooperator left to adopt the defective strategy. Because always 
Frank Schweitzer, Laxmidhar Behera, Heinz Mühlenbein:

Evolution of Cooperation in a Spatial Prisoner's Dilemma

Advances in Complex Systems, vol. 5, no. 2-3, pp. 269-299

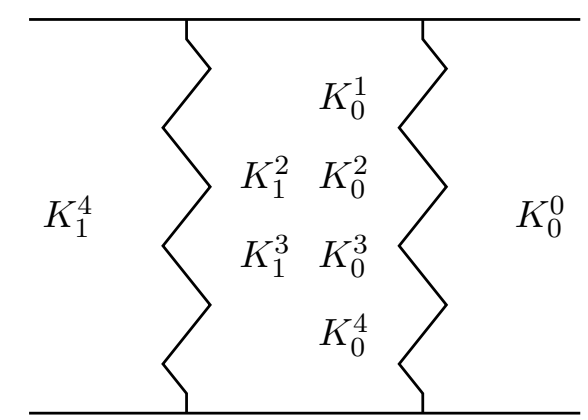

Figure 4: Sketch of the possible neighboring configurations in a border region between domains of cooperators $\left(K_{1}^{4}\right)$ and defectors $\left(K_{0}^{0}\right)$.

Table 1: Invasion of configurations $K_{0}^{s}$ "owned" by defectors into configurations $K_{1}^{s}$ "owned" by cooperators in the border region, Fig. 4 .

\begin{tabular}{ccl}
\hline \hline configuration & necessary conditions & stationary state \\
\hline$K_{0}^{1} \Rightarrow K_{1}^{2}$ & $a_{0}^{1}>a_{1}^{4}$ & complete invasion \\
$K_{0}^{1} \Rightarrow K_{1}^{3}$ & & \\
\hline$K_{0}^{2} \Rightarrow K_{1}^{2}$ & $a_{0}^{2}>a_{1}^{4}$ & unstable \\
$K_{0}^{2} \Rightarrow K_{1}^{3}$ & $a_{1}^{4}>a_{0}^{1}$ & coexistence (d) \\
\hline$K_{0}^{3} \Rightarrow K_{1}^{2}$ & $a_{0}^{3}>a_{1}^{4}$ & \\
$K_{0}^{3} \Rightarrow K_{1}^{3}$ & $a_{0}^{2}<a_{1}^{3}$ & coexistence (b) \\
& $a_{0}^{1}<a_{1}^{2}$ & \\
\hline \hline
\end{tabular}

$a_{0}^{4}>a_{0}^{3}>a_{0}^{2}>a_{0}^{1}$, eq. (17), this leads to the necessary condition for the complete invasion of defectors into the domain of cooperators:

$$
a_{0}^{1}>a_{1}^{4}
$$

The two remaining cases that lead to coexistence will be explained together with the next table, as well as the special case of $K_{0}^{4}$.

For the case (ii), invasion of configurations $K_{1}^{s}$ "owned" by cooperators into configurations $K_{0}^{s}$ "owned" by defectors, the results for the possible cases are listed in Table 2 . First, we notice that a complete invasion of cooperators into the whole population is not possible, because the necessary condition $a_{1}^{1}>a_{0}^{4}$, i.e. the lowest possible payoff for cooperators is larger than the highest possible payoff for defectors, is never fulfilled. Further, from eq. (18) we realize that configuration $K_{0}^{s}$ cannot be invaded by $K_{1}^{s}$ because the former payoff is always higher. But configuration $K_{1}^{s}$ could always 
Frank Schweitzer, Laxmidhar Behera, Heinz Mühlenbein:

Evolution of Cooperation in a Spatial Prisoner's Dilemma

Advances in Complex Systems, vol. 5, no. 2-3, pp. 269-299

Table 2: Invasion of configurations $K_{1}^{s}$ "owned" by cooperators into configurations $K_{0}^{s}$ "owned" by defectors in the border region, Fig. 4 .

\begin{tabular}{ccl}
\hline \hline configuration & necessary conditions & stationary state \\
\hline$K_{1}^{2} \Rightarrow K_{0}^{1}$ & $a_{1}^{2}>a_{0}^{1}$ & coexistence (a) \\
$K_{1}^{3} \Rightarrow K_{0}^{1}$ & $a_{1}^{3}>a_{0}^{2}$ & C majority \\
$K_{1}^{3} \Rightarrow K_{0}^{2}$ & $a_{1}^{4}>a_{0}^{3}$ & stationary domains \\
\hline$K_{1}^{2} \Rightarrow K_{0}^{1}$ & $a_{1}^{2}>a_{0}^{1}$ & coexistence (b) \\
$K_{1}^{3} \Rightarrow K_{0}^{1}$ & $a_{1}^{3}>a_{0}^{2}$ & C minority \\
$K_{1}^{3} \Rightarrow K_{0}^{2}$ & $a_{1}^{4}<a_{0}^{3}$ & spatial chaos \\
\hline \multicolumn{5}{c}{$a_{0}^{2}>a_{1}^{3}>a_{0}^{1}$} & coexistence (c) \\
$K_{1}^{3} \Rightarrow K_{0}^{1}$ & $a_{0}^{3}>a_{1}^{4}>a_{0}^{2}$ & C minority \\
& & small clusters \\
\hline$K_{1}^{2} \Rightarrow K_{0}^{2}$ & & \\
$K_{1}^{2} \Rightarrow K_{0}^{3}$ & not possible & \\
$K_{1}^{2} \Rightarrow K_{0}^{4}$ & & \\
$K_{1}^{3} \Rightarrow K_{0}^{3}$ & & \\
$K_{1}^{3} \Rightarrow K_{0}^{4}$ & & \\
\hline \hline
\end{tabular}

invade a neighboring configuration $K_{0}^{s-1}$ if the condition

$$
a_{1}^{s}>a_{0}^{s-1} \quad(s \in\{2,3,4\})
$$

would be satisfied. As eq. (16) shows, this is not always the case, but provided suitable values of $T$ and $P$, it can be possible. Thus, $K_{1}^{2}$ could invade a neighboring configuration $K_{0}^{1}$, and $K_{1}^{3}$ could invade both $K_{0}^{2}$ and $K_{0}^{1}$. However, we recall that $K_{1}^{3}$ and $K_{1}^{2}$ cannot invade $K_{0}^{3}$ because of eq. (18). This means that the invasion of cooperation necessarily stops at some point.

On the other hand, $K_{0}^{3}$ and could invade $K_{1}^{3}$ and $K_{1}^{2}$ because of eq. (18). But this kind of invasion does not happen if eq. (20) is satisfied. This can be explained by looking at Fig. 5 where the payoffs in the border region are shown under the assumption that eq. (20) is valid. In the example shown, the payoff of configuration $K_{0}^{3}$ is higher than $K_{1}^{2}$ or $K_{1}^{3}$ because of eq. (18). But because of eq. (20), $a_{1}^{4}>a_{0}^{3}$, thus the invasion of $K_{0}^{3}$ cannot take place, i.e. the border remains at its current position. A similar explanation holds for the remaining case $K_{0}^{2}$.

In conclusion, one (strong) condition for coexistence is given by eq. (20). For $s \in\{2,3,4\}$ this means basically three different inequalities. If all of them are satisfied, the cooperators become the global majority, because they are always allowed to invade $K_{0}^{1}, K_{0}^{2}$, whereas defectors in $K_{0}^{3}$ who could possibly invade the cooperation domain are stopped, as explained above. We could relax 
Frank Schweitzer, Laxmidhar Behera, Heinz Mühlenbein:

Evolution of Cooperation in a Spatial Prisoner's Dilemma

Advances in Complex Systems, vol. 5, no. 2-3, pp. 269-299

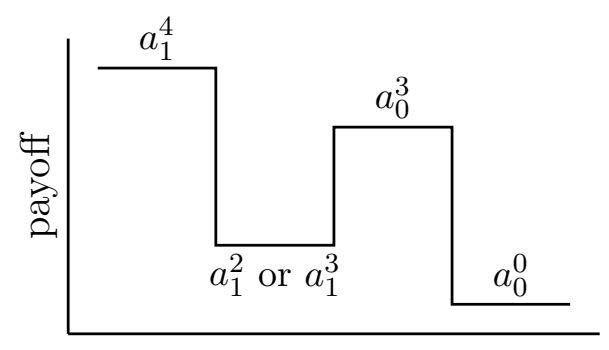

Figure 5: Payoffs in the border region between domains of cooperators $\left(K_{1}^{4}\right)$ and defectors $\left(K_{0}^{0}\right)$ with the assumption that eq. (20) is valid. It explains that configuration $K_{0}^{3}$ cannot invade neighboring configurations $K_{1}^{2}$ or $K_{1}^{3}$ because of the higher payoff of configuration $K_{1}^{4}$ that "backs" them from the other side.

the condition of eq. (20) that causes this stop. So, let us assume the case that only two of the coexistence inequalities are satisfied, i.e.

$$
a_{1}^{s}>a_{0}^{s-1}(s \in\{2,3\}) ; \quad a_{1}^{4}<a_{0}^{3}
$$

Then, the defector configuration $K_{0}^{3}$ can invade the cooperation configurations $K_{1}^{2}$, $K_{1}^{3}$, which eventually leads to the domination of defectors, i.e. the cooperators become a global minority. We note that in this case we will not find stable coexisting domains, but a nonstationary pattern that appears to be spatial chaos, as also shown in the next section.

If the strong condition for coexistence, eq. (20) is not satisfied, a weaker condition for coexistence could still hold true:

$$
a_{0}^{s-1}>a_{1}^{s}>a_{0}^{s-2}(s \in\{3,4\})
$$

This condition would lead to an even greater domination of defectors, because only the configuration $K_{0}^{1}$ can be invaded by cooperators, while all other ones are protected from this. In this case, as we will also show by means of computer simulations, the cooperators will only survive in very small clusters.

A very special case arises if none of the above coexistence inequalities are satisfied, but instead either one of the following conditions holds:

$$
\begin{array}{r}
a_{0}^{1}<a_{1}^{4}<a_{0}^{2} \\
a_{0}^{2}<a_{1}^{4} ; \quad a_{1}^{3}<a_{0}^{1} \\
a_{0}^{3}<a_{1}^{4} ; \quad a_{1}^{2}<a_{0}^{1}<a_{1}^{3}
\end{array}
$$

In this case, if we start from the special initial condition of only two domains separated by a planar interface, we find that this is also a stable configuration. But any deviation from this initial condition will eventually lead to an invasion of defectors. In particular, if we start from a random 
Frank Schweitzer, Laxmidhar Behera, Heinz Mühlenbein:

Evolution of Cooperation in a Spatial Prisoner's Dilemma

Advances in Complex Systems, vol. 5, no. 2-3, pp. 269-299

initial distribution of cooperators and defectors, invasion of defectors will always take place under one of the conditions definded by eqs. (23)-(25). Therefore, this case has been named unstable coexistence (d) in Table 1. It denotes the transition from the case of complete invasion of defectors to the case of stable coexistence between cooperators and defectors.

The only remaining case to be explained is $K_{0}^{4}$ in the border region, which arises if cooperation invades into the domain of defection from different sides. Then, it can happen that a single defecting agent is trapped within the domain of cooperators. Because of $a_{0}^{4}>a_{1}^{4}$, defection will invade all neighboring sites during the next generation, this way leading to a configuration $K_{0}^{0}$ in the border region. This could lead to a growth of the domain of defectors if the conditions for complete invasion, eq. (19) is satisfied (see also Table 11). But if these conditions are not satisfied, there are various possibilities dependent on whether three, two or one of the inequalities for coexistence are satisfied (see also Table 2). $K_{0}^{0}$ is surrounded by other configurations $K_{0}^{1}, K_{0}^{2}$ or $K_{0}^{3}$ in the larger neighborhood. If eq. (20) is valid and only $K_{0}^{1}, K_{0}^{2}$ are present, then in the next generation they will be occupied by cooperators, as explained above, leaving the defector in the central cell in its trapped situation, again. So, a cycle of $K_{0}^{4}$ and $K_{0}^{0}$ alternating is created. If $K_{0}^{3}$ is present, this will create a stable border between the spatial domains of cooperators, this way leading to coexistence

as explained above, too. If eq. (21) is valid and $K_{0}^{3}$ is present, this will lead to the split-up of the domains of cooperators. If eq. (22) is valid and $K_{0}^{3}$ is present, this will lead to a growth of the defector domain.

In the following section, we will apply the analytical conditions derived for the invasion and coexistence of cooperators and defectors to find out critical values of the payoff matrix that may lead to the predicted spatial patterns.

\section{Critical payoff values for invasion and coexistence}

\subsection{Derivation of a phase diagram}

The investigations of the previous section have provided us with a set of inequalities for the payoffs $a_{\theta}^{s}$, in order to find certain patterns of invasion and coexistence of cooperators and defectors. The possible payoffs $a_{\theta}^{s}$, eq. (16) depend on the set of variables $\{R ; S ; T ; P\}$ of the payoff matrix, eq. (4). In the following, we fix the values for $R=3$ and $S=0$, i.e. the payoffs for a cooperating agent $i$, to the standard values, and this way derive the critical conditions for the payoffs for a defecting agent, $T$ and $P$. With the known payoffs $a_{\theta}^{s}$, eq. (16), we find from the different inequalities (20) 
the following set of conditions:
(a) $a_{1}^{4} \lessgtr a_{0}^{3} \Rightarrow 3 T+P=12$
(b) $a_{1}^{3} \lessgtr a_{0}^{2} \Rightarrow 2 T+2 P=9$
(c) $a_{1}^{2} \lessgtr a_{0}^{1} \quad \Rightarrow \quad T+3 P=6$
(d) $a_{1}^{4} \lessgtr a_{0}^{2} \quad \Rightarrow \quad 2 T+2 P=12$
(e) $a_{1}^{3} \lessgtr a_{0}^{1} \quad \Rightarrow \quad T+3 P=9$
(f) $a_{1}^{4} \lessgtr a_{0}^{1} \Rightarrow T+3 P=12$

Additionally, we always have

$$
3 \leq T \leq 6 ; \quad 0 \leq P \leq 3
$$

because of the ineqs. (5), (6). With these restrictions, we are able to plot a phase diagram in the $\{P, T\}$ parameter space, Fig. 6 where the conditions (26) mark the different boundaries between the phases. The labels A-E in Fig. 6 denote the parameter regions associated with the different stationary patterns and shall be explained in detail in the following.

\subsection{Complete invasion of defectors (region E)}

For the complete invasion of defectors into the domain of cooperators, the necessary condition of eq. (19) applies, i.e.

$$
\frac{T+3 P}{4}>R
$$

With the fixed values for $R$ and $P$ and eq. (27) we find

$$
12-3 P<T<6 \quad \text { if } \quad 2<P<3
$$

This condition defines region $\mathbf{E}$ in the parameter space shown in Fig. 6. Thus, for the appropriate $\{T, P\}$ values the stationary state is always entirely dominated by the defectors regardless of the initial conditions, therefore no further computer simulations are presented here.

\subsection{Coexistence with a majority of cooperators (region A)}

Coexistence between cooperators and defectors becomes possible if eq. (20) is fulfilled. As we have shown in Sect. 3, this leads to different cases dependent on how many inequalities of eq. (20) are satisfied. If all of them are fulfilled, this leads to the conditions:

$$
\frac{2 R+2 S}{4}>\frac{T+3 P}{4} ; \quad \frac{3 R+S}{4}>\frac{2 T+2 P}{4} ; \quad R>\frac{3 T+P}{4}
$$




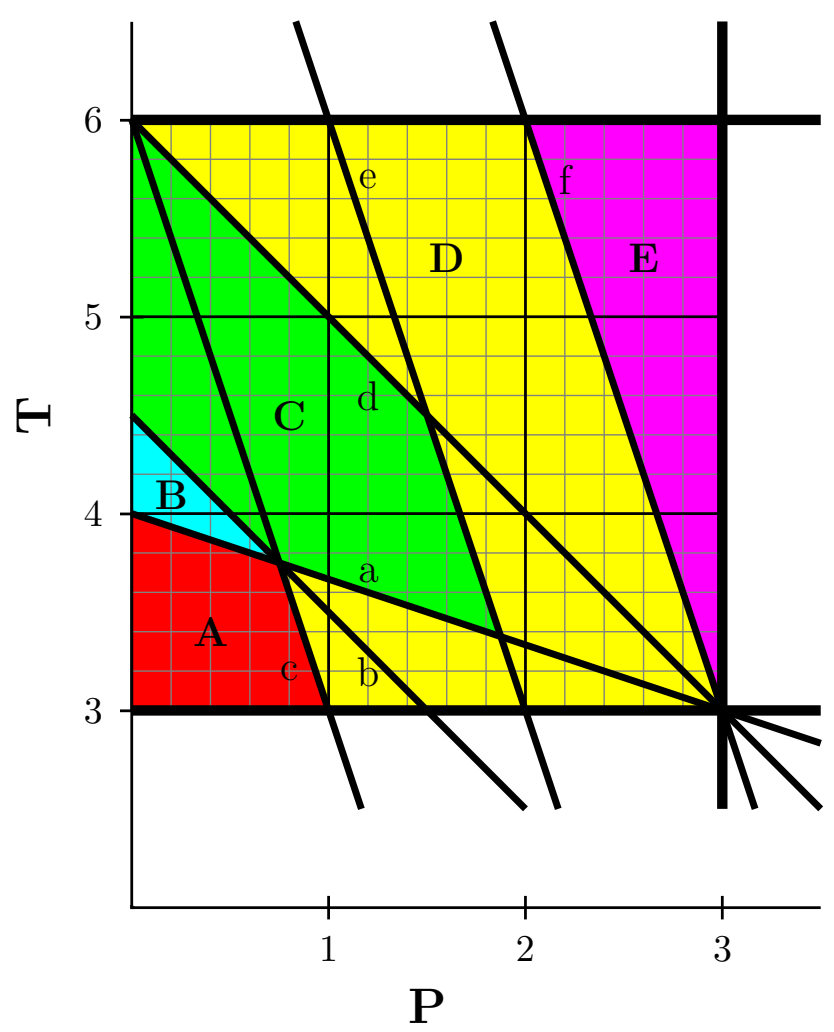

Figure 6: Phase diagram in the $\{T, P\}$ parameter space for fixed values of $R=3$ and $S=0$ of the payoff matrix, eq. (田). The different lines result from eq. (26) $(a-f)$ and eq. (27). The areas $A-E$ (in different gray scales) indicate parameter regions that lead to particular spatial patterns in the stationary limit. For a detailed explanation see text. Note that the "classical" parameter values $\{R ; S ; T ; P\}=\{3 ; 0 ; 5 ; 1\}$ are just on the border between regions $C$ and $D$.

With the fixed values for $R$ and $S$ we find:

$$
T+3 P<6 ; \quad 2 T+2 P<9 ; \quad 3 T+P<12
$$

where additionally eq. (27) applies. The solution is then given by:

$$
\begin{array}{lllrl}
3 & <T<4-\frac{P}{3} & \text { if } & 0 & <P<0.75 \\
3 & <T<6-3 P & \text { if } & 0.75 & <P<1.0
\end{array}
$$

These two conditions define region A of Fig. 6. Eq. (33) gives the respective payoffs $a_{\theta}^{s}$, eq. (16) for the choosen set of parameters, $\{R ; S ; T ; P\}=\{3.0 ; 0.0 ; 3.5 ; 0.5\}$. The $a_{\theta}^{s}$ can be used to verify the 
Frank Schweitzer, Laxmidhar Behera, Heinz Mühlenbein:

Evolution of Cooperation in a Spatial Prisoner's Dilemma

Advances in Complex Systems, vol. 5, no. 2-3, pp. 269-299

general considerations of Sect. 3. They are printed here to simplify the comparison.

$\begin{array}{cccccccccc}a_{1}^{4} & a_{1}^{3} & a_{1}^{2} & a_{1}^{1} & a_{1}^{0} & a_{0}^{4} & a_{0}^{3} & a_{0}^{2} & a_{0}^{1} & a_{0}^{0} \\ 3.0 & 2.25 & 1.5 & 0.75 & 0.0 & 3.5 & 2.75 & 2.0 & 1.25 & 0.5\end{array}$

Note that the $a_{1}^{s}$ are the same as in the "classical" case, eq. (16) since they only depend on the values of $R$ and $S$ that are not changed. For the appropriate $\{T, P\}$ values the stationary state always shows a coexistence between cooperators and defectors where the cooperators are a majority. Note, that this parameter region is just opposite to the region E, Fig. 6 where the stationary state is characterized by an extinction of the cooperators.

The coexistence between cooperators and defectors shall be also demonstrated by means of computer simulations. To elucidate the dynamics, we have choosen two different initial conditions for the simulations, shown in Fig. 7. The left part of Fig. 7 shows a regular situation of only one cluster of cooperators is a sea of defectors, i.e. the cooperators are the absolute minority. For this initial condition, Fig. 9 shows the evolution of the global frequency for the different parameter regions $\mathbf{A}$, $\mathbf{B}$ and $\mathbf{C}$. The right part of Fig. 7 shows a random initial distribution of cooperators and defectors with the same initial frequency, $f(0)=0.5$. In order to study the influence of the initial frequency on the long-term dynamics, we will also choose random distributions with different values of $f(0)$.
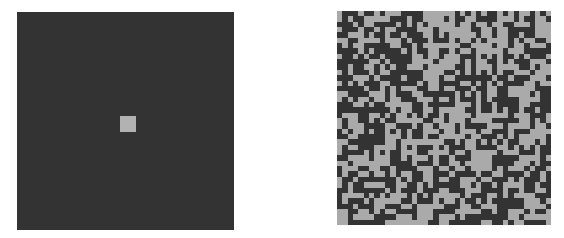

Figure 7: Initial spatial distributions of cooperators (grey) and defectors (black) on a CA of size $N=40 \times 40$, used for the computer simulations: (left) One cluster of 9 cooperators, $f(0)=0.0056$, (right) random spatial distribution of cooperators and defectors, shown for $f(0)=0.5$.
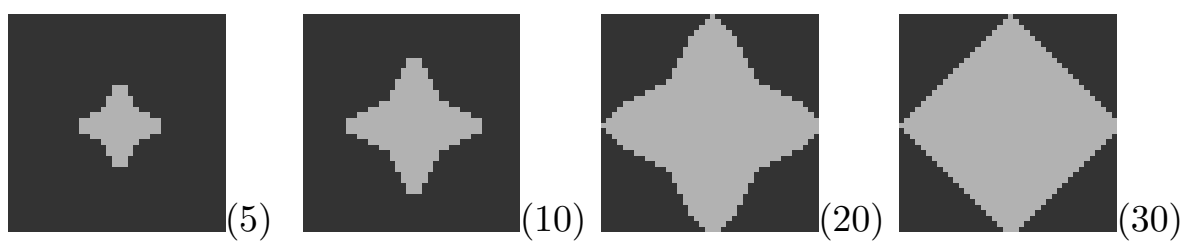

Figure 8: Time series of the spatial distribution of cooperators (grey) and defectors (black) on a CA of size $N=40 \times 40$. The time is given by the numbers of generations in brackets. Initial condition: One cluster of 9 cooperators, Fig. [(left). Parameters for the payoff matrix, eq. (4): $\{R ; S ; T ; P\}=\{3.0 ; 0.0 ; 3.5 ; 0.5\}$ (region $\mathrm{A})$. 
Frank Schweitzer, Laxmidhar Behera, Heinz Mühlenbein:

Evolution of Cooperation in a Spatial Prisoner's Dilemma

Advances in Complex Systems, vol. 5, no. 2-3, pp. 269-299

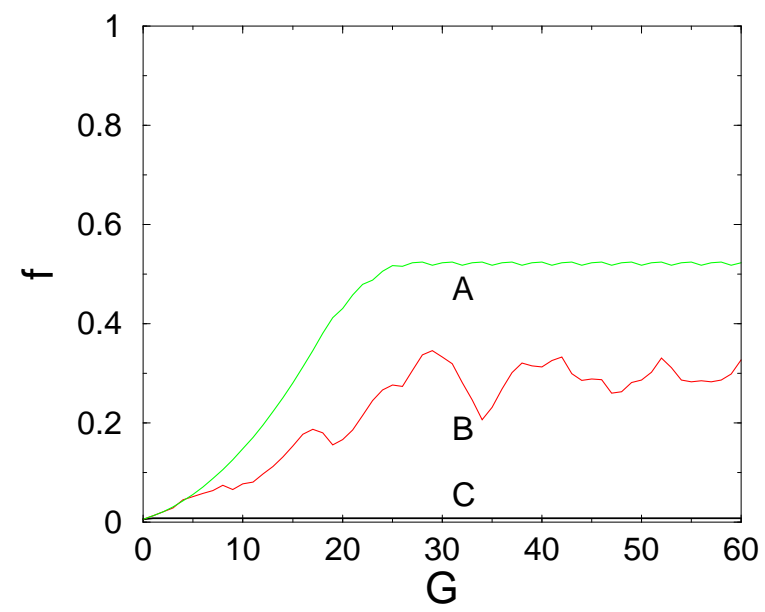

Figure 9: Global frequency of cooperators, $f$, eq. (11), vs. time (number of generations). Initial condition: One cluster of 9 cooperators, Fig. 母(left), $f(0)=0.0056$. Parameters for the payoff matrix, eq. (鸟), $\{R ; S ; T ; P\}:$ (A) $\{3.0 ; 0.0 ; 3.5 ; 0.5\}$ (region A), (B) $\{3.0 ; 0.0 ; 3.9 ; 0.5\}$ (region B), (C) $\{3.0 ; 0.0 ; 4.5 ; 1.0\}$ (region $\mathrm{C}$ ).
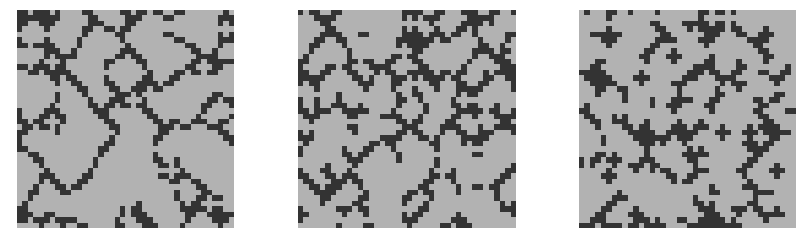

Figure 10: Final (steady state) spatial distribution of cooperators (grey) and defectors (black) on a CA of size $N=40 \times 40$. Initial condition: random spatial distribution of cooperators and defectors, left: $f(0)=0.5$, middle: $f(0)=0.75$, right: $f(0)=0.9$. Parameters: $\{R ; S ; T ; P\}=$ $\{3.0 ; 0.0 ; 3.5 ; 0.5\}$ (region A).

From the computer simulations shown in Fig. 2 and Figs. 8, 11, we can draw the following conclusions in agreement with the general discussion in Sect. 3 and the discussion of Fig. 2 in Sect. 2.2: We observe the formation of spatial domains of cooperators that are separated by narrow regions of defectors. Only the special case of a singular initial cluster shown in Fig. 8 leads to a regular stationary final pattern where cooperators and defectors are clearly separated into two domains. In the stationary state we find a stable coexistence between cooperators and defectors. The cooperators become the majority $(f>0.5)$ almost independent of their initial spatial configuration and initial frequency as shown in Fig. 9A and Fig. 11. The stationary frequency is almost constant (with very small periodic changes). 
Frank Schweitzer, Laxmidhar Behera, Heinz Mühlenbein:

Evolution of Cooperation in a Spatial Prisoner's Dilemma

Advances in Complex Systems, vol. 5, no. 2-3, pp. 269-299

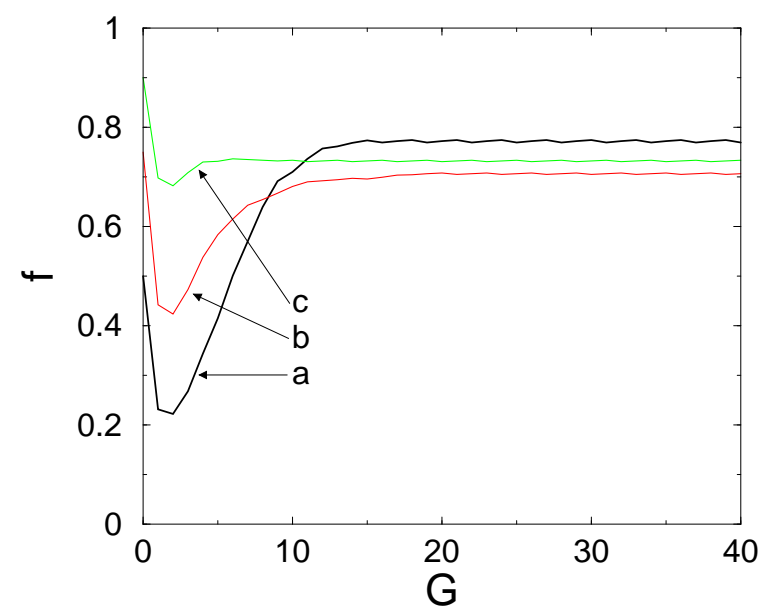

Figure 11: Global frequency of cooperators, $f$, eq. (仼), vs. time (number of generations). The data have been obtained from simulations with three different initial frequencies: (a) $f(0)=0.5$, (b) $f(0)=0.75$, (c) $f(0)=0.9$. See also Fig. 10 for the final spatial distributions. Parameters: $\{R ; S ; T ; P\}=\{3.0 ; 0.0 ; 3.5 ; 0.5\}$ (region A).

\subsection{Coexistence with a minority of cooperators - spatial chaos (region B)}

If only two of the coexistence conditions are satisfied, i.e. eq. (21) applies, this leads to:

$$
\frac{2 R+2 S}{4}>\frac{T+3 P}{4} ; \quad \frac{3 R}{4}>\frac{2 T+2 P}{4} ; \quad R<\frac{3 T+P}{4}
$$

With the fixed values for $R$ and $S$, we find:

$$
T+3 P<6 ; \quad 2 T+2 P<9 ; \quad 3 T+P>12
$$

With eq. (27), this eventually results in the solution:

$$
4-\frac{P}{3}<T<4.5-P \quad \text { if } \quad 0<P<0.75
$$

This condition defines region $\mathbf{B}$ of Fig. 6. Eq. (37) gives the respective payoffs $a_{\theta}^{s}$, eq. (16) for the choosen set of parameters, $\{R ; S ; T ; P\}=\{3.0 ; 0.0 ; 3.9 ; 0.5\}$. The $a_{\theta}^{s}$ can be used to verify the general considerations of Sect. 3 .

$$
\begin{array}{cccccccccc}
a_{1}^{4} & a_{1}^{3} & a_{1}^{2} & a_{1}^{1} & a_{1}^{0} & a_{0}^{4} & a_{0}^{3} & a_{0}^{2} & a_{0}^{1} & a_{0}^{0} \\
3.0 & 2.25 & 1.5 & 0.75 & 0.0 & 3.9 & 3.05 & 2.2 & 1.35 & 0.5
\end{array}
$$

For the appropriate $\{T, P\}$ values, there is always a nonstationary coexistence between cooperators and defectors where the cooperators are a minority. This is also shown for the two different initial conditions, Fig. 团 in the computer simulations of Fig. 12, Fig. 13, Fig. 9B and Fig. 14. 

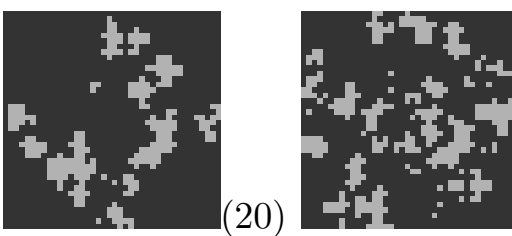

$(30)$

Figure 12: Time series of the spatial distribution of cooperators (grey) and defectors (black) on a CA of size $N=40 \times 40$. The time is given by the numbers of generations in brackets. Initial condition: One cluster of 9 cooperators, Fig. 7(left). Parameters for the payoff matrix, eq. (4): $\{R ; S ; T ; P\}=\{3.0 ; 0.0 ; 3.9 ; 0.5\}$ (region $\mathrm{B}$ ).
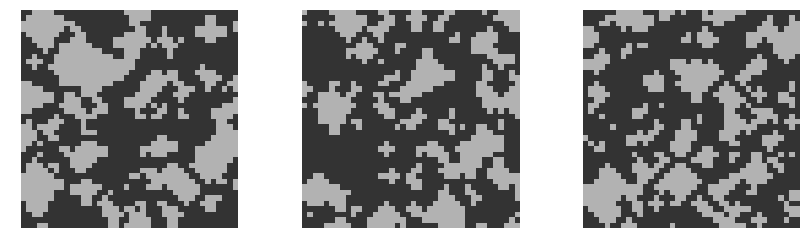

Figure 13: Spatial distribution of cooperators (grey) and defectors (black) on a CA of size $N=$ $40 \times 40$ after $G=100$ generations. Note, that there is no stationary pattern asymptotically. Initial condition: random spatial distribution of cooperators and defectors, left: $f(0)=0.5$, middle: $f(0)=$ 0.75 , right: $f(0)=0.9$. Parameters: $\{R ; S ; T ; P\}=\{3.0 ; 0.0 ; 3.9 ; 0.5\}$ (region B).

The time series of Fig. 12 - that should be compared to Fig. 8 for parameter region $\mathbf{A}$ - shows that the initial cluster of cooperators first grows, but then splits up into smaller clusters, which grow, split up again and may dissappear. This leads to a non-stationary spatial distribution even in the long run. In fact, it has been already argued by Nowak [32] that this regime can be characterized as spatiotemporal chaos. If we look for the time dependence of the global frequency in this case, Fig. 9B and Fig. 14, we find no convergence to a (quasi)stationary value as in Fig. 11, but rather large variations over time. Noteworthy, in the average the global frequency of cooperators is below 0.5, i.e. they are indeed the minority almost independent of the initial frequencies. Further, we note that the cooperators still form large clusters, as shown Fig. 13 that can be compared to Fig. 10 for region $\mathbf{A}$.

\subsection{Coexistence with cooperators in small clusters (region $\mathrm{C}$ )}

Eventually, we also show examples for the weaker coexistence condition, eq. (22). In this case, the conditions are given as:

$$
\frac{2 T+2 P}{4}>\frac{3 R+S}{4}>\frac{T+3 P}{4} ; \quad \frac{3 T+P}{4}>R>\frac{2 T+2 P}{4}
$$


Frank Schweitzer, Laxmidhar Behera, Heinz Mühlenbein:

Evolution of Cooperation in a Spatial Prisoner's Dilemma

Advances in Complex Systems, vol. 5, no. 2-3, pp. 269-299

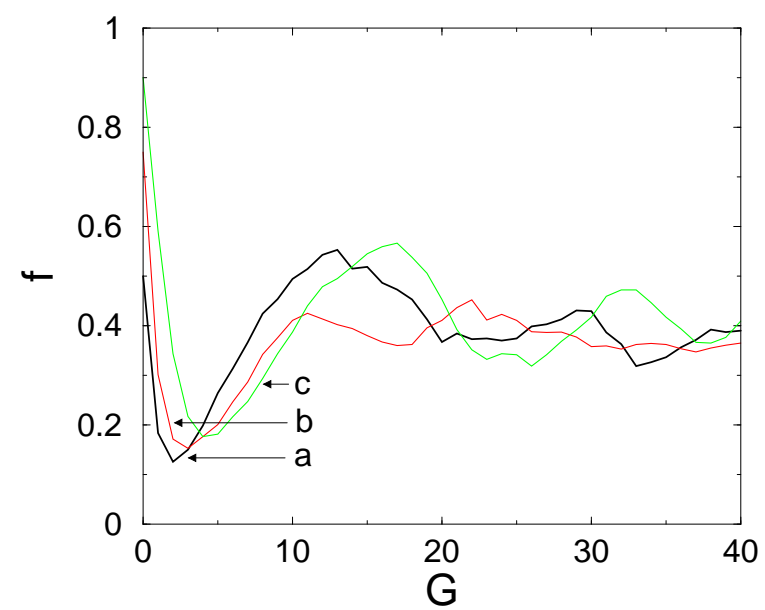

Figure 14: Global frequency of cooperators, $f$, eq. (四), vs. time (number of generations). The data have been obtained from simulations with three different initial frequencies: (a) $f(0)=0.5$, (b) $f(0)=0.75$, (c) $f(0)=0.9$. See also Fig. 13 for the final spatial distributions. Parameters: $\{R ; S ; T ; P\}=\{3.0 ; 0.0 ; 3.9 ; 0.5\}$ (region $\mathrm{B}$ ).

With $R=3$ and $S=0$, this results in:

$$
T+3 P<9 ; \quad 2 T+3 P<12 ; \quad 2 T+2 P>9 ; \quad 3 T+P>12
$$

where additionally eq. (27) applies. The solution of eq. (39) is then given by:

$$
\begin{array}{lllrl}
4-P / 3 & <T<9-3 P & \text { if } & 1.5 & <P<1.875 \\
4-P / 3 & <T<6-P & \text { if } & 0.75 & <P<1.5 \\
4.5-P & <T<6-P & \text { if } & 0.0 & <P<0.75
\end{array}
$$

These three conditions define region $\mathbf{C}$ of Fig. 6. Eq. (41) gives the respective payoffs $a_{\theta}^{s}$, eq. (16) for the choosen set of parameters, $\{R ; S ; T ; P\}=\{3.0 ; 0.0 ; 4.5 ; 1.0\}$. The $a_{\theta}^{s}$ can be used to verify the general considerations of Sect. 3 .

$$
\begin{array}{cccccccccc}
a_{1}^{4} & a_{1}^{3} & a_{1}^{2} & a_{1}^{1} & a_{1}^{0} & a_{0}^{4} & a_{0}^{3} & a_{0}^{2} & a_{0}^{1} & a_{0}^{0} \\
3.0 & 2.25 & 1.5 & 0.75 & 0.0 & 4.5 & 3.625 & 2.75 & 1.875 & 1.0
\end{array}
$$

For the appropriate $\{T, P\}$ values, the stationary states are characterized by a stable coexistence between cooperators and defectors where the cooperators survive only in small clusters, while the defectors dominate. This is also shown for the two different initial conditions of Fig. 7 in the computer simulation of Fig. 15, Fig. 16, Fig. 9C and Fig. 17.

Fig. 15 - that should be compared to Fig. 8 for parameter region $\mathbf{A}$ and Fig. 12 for region $\mathbf{B}$ indicates that the initial cluster grows only very little in size, i.e. from 9 to 13 cooperators, and then 

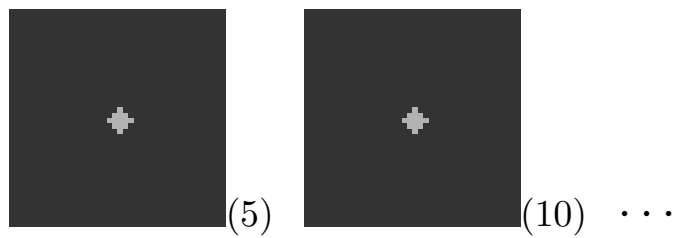

Figure 15: Time series of the spatial distribution of cooperators (grey) and defectors (black) on a CA of size $N=40 \times 40$. The time is given by the numbers of generations in brackets. Initial condition: One cluster of 9 cooperators, Fig. $\mathbb{W}($ left). Parameters for the payoff matrix, eq. (4): $\{R ; S ; T ; P\}=\{3.0 ; 0.0 ; 4.5 ; 1.0\}$ (region $\mathrm{C}$ ).
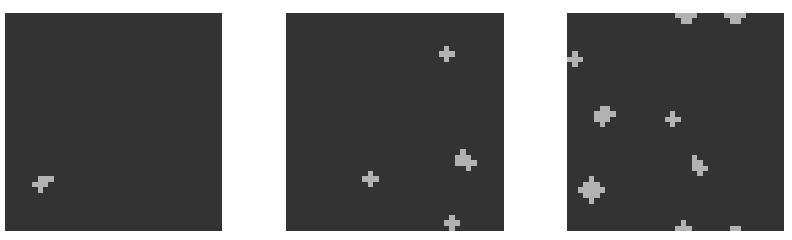

Figure 16: Final (steady state) spatial distribution of cooperators (grey) and defectors (black) on a CA of size $N=40 \times 40$. Initial condition: random spatial distribution of cooperators and defectors, left: $f(0)=0.5$, middle: $f(0)=0.75$, right: $f(0)=0.9$. Parameters: $\{R ; S ; T ; P\}=$ $\{3.0 ; 0.0 ; 4.5 ; 1.0\}$ (region $\mathrm{C}$ ).

remains stable at its borders. However, if the initial configuration would have more than one cluster, then not all of them may survive. If two cooperating clusters are very near, then the configuration $K_{0}^{4}$ may appear between them, which leads to an invasion of the defectors in the next step and the further discussion of Sect. 3.2 applies. Thus, several small clusters may only survive if there is a certain distance between them. This can be also seen in Fig. 16 that shows the stationary spatial distributions for parameter region $\mathbf{C}$, in comparison to Fig. 10 for region $\mathbf{A}$ and Fig. 13 for region B. We observe only very small clusters at a certain distance, the number of which further depend on the initial frequency of cooperators. The evolution of the global frequency, Fig. 9C and Fig. 17, also clearly shows that the cooperators survive only as a small minority, which should be compared to Fig. 11 and Fig. 14.

\subsection{Special case of unstable coexistence (region D)}

The last case to be discussed is the special case of eq. (23)-(25), which leads to coexistence only for very special initial conditions, as discussed in Sect. 3.2. From eq. (23), we find:

$$
\frac{T+3 P}{4}<R<\frac{2 T+2 P}{4}
$$




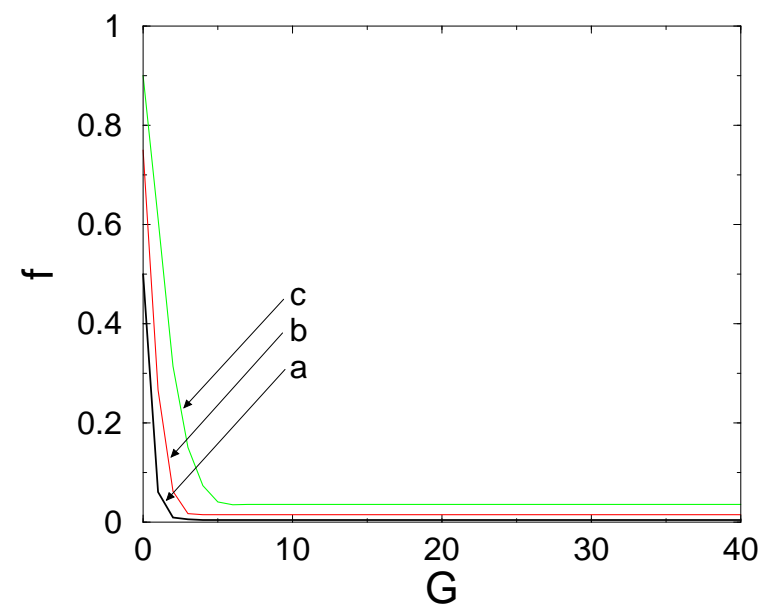

Figure 17: Global frequency of cooperators, $f$, eq. (何), vs. time (number of generations). The data have been obtained from simulations with three different initial frequencies: (a) $f(0)=0.5$, (b) $f(0)=0.75$, (c) $f(0)=0.9$. See also Fig. 16 for the final spatial distributions. Parameters: $\{R ; S ; T ; P\}=\{3.0 ; 0.0 ; 4.5 ; 1.0\}$ (region $\mathrm{C}$ ).

With $R=3, S=0$, we have following conditions:

$$
T<12-3 P ; \quad T<6-P
$$

where additionally eq. (27) applies. This leads to the solution:

$$
6-P<T<6 \text { if } 0<P<3
$$

Similarly, the special case of eq. (24) with $R=3, S=0$ and eq. (27) leads to:

$$
\begin{array}{ll}
\frac{2 T+2 P}{4}<R \quad ; & \frac{3 R+S}{4}<\frac{T+3 P}{4} \\
2 T+2 P<12 \quad ; & 9<T+3 P
\end{array}
$$

with the solution:

$$
3<T<6-P \quad \text { if } \quad 2<P<3
$$

while the special case of eq. (25) results in:

$$
\begin{array}{ll}
\frac{3 T+P}{4}<R \quad ; \quad & \frac{2 R+2 S}{4}<\frac{T+3 P}{4}<\frac{3 R+S}{4} \\
3 T+P<12 \quad ; \quad & 6<T+3 P<9
\end{array}
$$

with the solution:

$$
\begin{array}{rlrrrr}
3 & <T<\frac{12-P}{3} & \text { if } & 1.0 & <P<2.0 \\
6-3 P & <T<\frac{12-P}{3} & \text { if } & 0.75 & <P<1.0
\end{array}
$$


Frank Schweitzer, Laxmidhar Behera, Heinz Mühlenbein:

Evolution of Cooperation in a Spatial Prisoner's Dilemma

Advances in Complex Systems, vol. 5, no. 2-3, pp. 269-299

The three solutions (44), (46), (48) together define parameter region $\mathbf{D}$ of Fig. 6. Provided the appropriate $\{T, P\}$ values, we observe in this case only an unstable coexistence of cooperators and defectors, i.e. for an random initial distribution the dynamics will always lead to an invasion of the defectors until a complete extinction of the cooperators - rather similar to the dynamics in the adjacent parameter region E. Only for the special case of two domains with a straight border, the coexistence remains stable.

Looking at the phase diagram of Fig. 6, we notice that region $\mathbf{D}$ separates the regions of stable coexistence (A-C) from the region of complete invasion of defectors $(\mathbf{E})$, i.e. D denotes the transition region between the two different stationary regimes, and the border between the two regions $\mathbf{C}$ and D just marks the transition from stability (i.e. stable coexistence) to instability (i.e. unstable coexistence). We further notice that the "classical" parameter values $\{R ; S ; T ; P\}=\{3 ; 0 ; 5 ; 1\}$ are just on the border between regions $\mathbf{C}$ and $\mathbf{D}$, i.e. for a random initial distribution the classical spatial 5-person game will always lead to the complete invasion of defectors and the extinction of cooperators, as already observed by means of computer simulations.

\subsection{Dynamics at the border regions}

So far, we have discussed the dynamics for the pure parameter regions, A-E. However, the question of interest is how the system behaves if we choose the parameters of the payoff matrix on the border between two such regions. We have already mentioned the case of the $\mathbf{C} \mid \mathbf{D}$ border, that marks the transition from (stable) coexistence to invasion. Fig. 18 presents results of computer simulations for the $\mathbf{A} \mid \mathbf{B}$ and $\mathbf{B} \mid \mathbf{C}$ border.
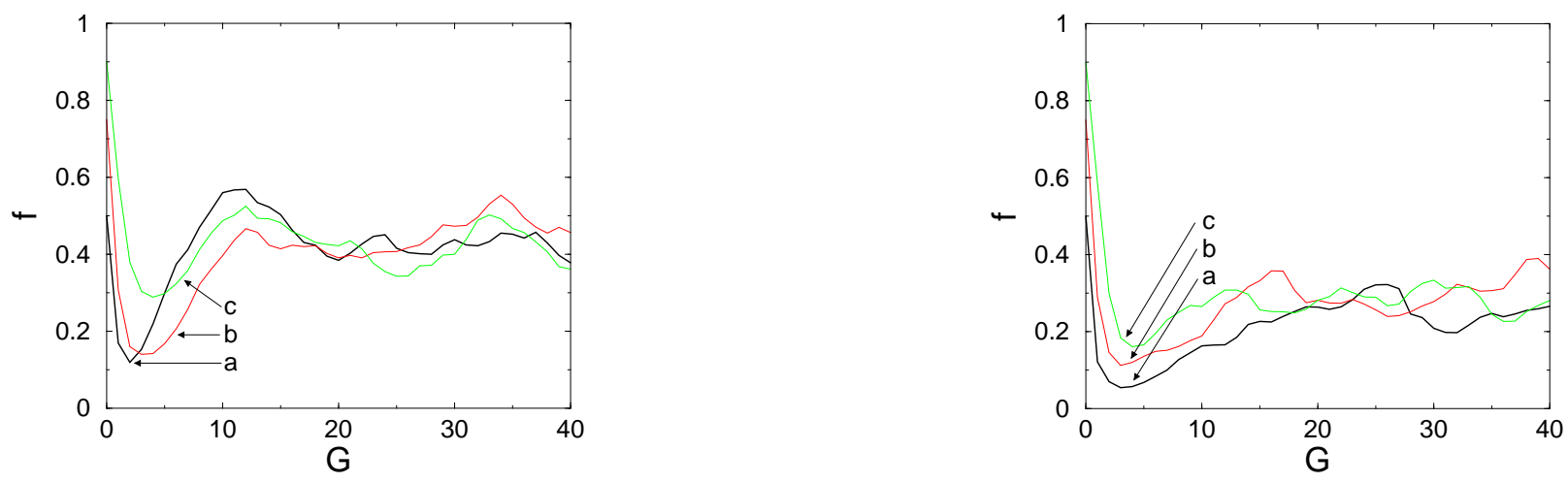

Figure 18: Global frequency of cooperators, $f$, eq. (11), vs. time (number of generations). The data have been obtained from simulations with three different initial frequencies: (a) $f(0)=0.5$, (b) $f(0)=0.75$, (c) $f(0)=0.9$. Parameters for $\{R ; S ; T ; P\}$ : (left) $\{3.0 ; 0.0 ; 3.833 ; 0.5\}$ (border between regions $\mathrm{A}$ and $\mathrm{B})$, (right) $\{3.0 ; 0.0 ; 4.0 ; 0.5\}$ (border between regions $\mathrm{B}$ and $\mathrm{C}$ ). 
Frank Schweitzer, Laxmidhar Behera, Heinz Mühlenbein:

Evolution of Cooperation in a Spatial Prisoner's Dilemma

Advances in Complex Systems, vol. 5, no. 2-3, pp. 269-299

As we see from the time evolution of the global frequencies, the dynamics in this case is basically the same as for region $\mathbf{B}$, i.e. there is no (quasi)stationary regime as for regions $\mathbf{A}$ or $\mathbf{C}$, and we may expect a spatiotemporal chaos again. Despite this, the average global frequency in the long run is also decreasing when going from region $\mathbf{A}$ to $\mathbf{C}$. In Fig. 18(left), i.e. on the border $\mathbf{A} \mid \mathbf{B}$ it is below the values of region $\mathbf{A}$, where the cooperators appear as a majority, Fig. 11, but above the values of region $\mathbf{B}$, where they already appear as a minority, Fig. 14. But in Fig. 18(right), i.e. on the border $\mathbf{B} \mid \mathbf{C}$ it is below the values of region $\mathbf{B}$, but still above the values of region $\mathbf{B}$, where the cooperators appear as a clear majority, Fig. 17.

\subsection{Nowak's results revisited}

For the parameter region $\mathbf{B}$ we already mentioned the existence of spatiotemporal chaos in the distribution of cooperators and defectors. This has been first observed by Nowak [32] by means of computer simulations. Based on our detailed theoretical investigations above, we are now able to derive the critical parameter values for Nowak's simulations.

Different from the standard values of the prisoner's dilemma, $\{R ; S ; T ; P\}=\{3 ; 0 ; 5 ; 1\}$, Nowak has used the following values for the payoff matrix:

$$
\{R ; S ; T ; P\}=\{1 ; 0 ; b ; 0\}
$$

i.e. all payoffs are fixed, while only the payoff for the defecting agent playing with a cooperating agent is an adjustable value, $b$. In order to fulfill the conditions for the payoff, eqs. (5), (6), $b$ can have only values of $1<b<2$. Further, we notice that the payoff matrix given by eq. (49) does not strictly fulfill the conditions of the prisoner's dilemma, because of $P=S$ in this case.

We can now apply the conditions derived for the different regions to the payoff matrix of eq. (49). The results are given in Table 3 .

Table 3: Parameter range of the payoff $b$, eq. (49) to obtain the different spatial patterns in the asymtotic state.

\begin{tabular}{llc}
\hline \hline stationary state & condition & range of $b$ values \\
\hline A: coexistence with large domains of cooperators & eq. (30) & $1.0<b<1.33$ \\
B: coexistence with spatial chaos & eq. $(34)$ & $1.33<b<1.5$ \\
C: coexistence with small clusters of cooperators & eq. (38) & $1.5<b<2.0$ \\
\hline \hline
\end{tabular}

We note again that these regions have been found in [32] by means of computer simulations, while we have confirmed the results based on an analytical investigation of the stability conditions. Further, 
Frank Schweitzer, Laxmidhar Behera, Heinz Mühlenbein:

Evolution of Cooperation in a Spatial Prisoner's Dilemma

Advances in Complex Systems, vol. 5, no. 2-3, pp. 269-299

we want to point out that the other two dynamical regimes, i.e. E: complete invasion of defectors and D: unstable coexistence, do not exist for the payoff matrix, eq. (49).

\section{Interactions in a larger neighborhood}

In this section, we want to implement the "exact" (or complete) 5-person game for the one-shot PD game on the square lattice - which is to our knowledge not investigated so far. Since the payoff matrix for the 5-person game has been computed in this paper using the concept of the 2-person game, Sect. 2.2, we will continue to explain the dynamics of the 5-person game in terms of 2-person games. So far, we have investigated the spatial 5-person game under the assumptions given in Sect. 2.2, namely (i) decomposition into independent, simultaneous 2-person games, (ii) each agent only plays with its $m$ nearest neighbors. This has reduced the game to $m$ 2-person games played by each agent.

In the following, we want to discuss a different variant of the game which instead of (ii) assumes that in a neighborhood of $n=5$ each agent plays a 2-person game with every other agent in this neighborhood. For a given neighborhood, this increases the number of 2-person games to $m \times n / 2=10$. We further have to take into account that agent $i$ itself is additionally part of $m$ different neighborhoods of size $n=5$ centered around its nearest neighbors at the positions $i_{j}(j=1, \ldots, m)$. This results in the consideration of a larger neighborhood of size $n=13$ that includes also the $r$ second nearest neighbors of agent $i$ (see Fig. 1). The total number of 2-person games played independently in this larger neighborhood is given by $m n^{2} / 2=50$, but we can easily verify that agent $i$ at position $j=0$ participates only in 20 of these. Specifically, he plays 4 games in his "own neighborhood" $(j=0)$ and 4 times 4 games in the neighborhoods of his neighbors $(j=1, \ldots, m)$. In these 20 games, he meets always twice (in two different 5 -person games) both with his $m$ nearest neighbors and with the neighboring agents at the positions $j=6,8,10,12$, but only once with the agents at the second nearest neighbor positions $j=5,7,9,11$ (cf. Fig. 11).

This in turn results in a different payoff, dependent on the number of cooperators and defectors in the larger neighborhood of agent $i$. Since the payoff is again calculated from the independent 2-person games, we can still use eq. (11) for $a_{i}$, but we have to recalculate the fractions $z_{i}^{1}$ and $z_{i}^{0}$ according to the modified game. I.e. eq. (10) has to be replaced by:

$$
z_{i}^{\theta}=\frac{1}{(m+r)}\left(2 \sum_{j=1}^{m+r} \delta_{\theta \theta_{i_{j}}}-\sum_{j \in 5,7,9,11} \delta_{\theta \theta_{i_{j}}}\right) ; z_{i}^{(1-\theta)}=1-z_{i}^{\theta} \quad(\theta \in\{0,1\})
$$

Eq. (50) can be interpreted in a way that agent $i$ - in addition to his nearest neighbors - now also interacts with his second nearest neighbors, but if they are not adjacent to his place (i.e. $j \in 5,7,9,11)$, this interaction occurs less frequently - or, the payoff is counted with a smaller weight, respectively. 
We do not intend to give here a detailed analysis of this modified case, the effect of the weighted interaction in the larger neighborhood shall be rather demonstrated by some computer simulations that allow a comparison to the previous results. For the dynamics of the modified game the update rule, eqs. (13), (14) still applies, i.e. agent $i$ adopts the $C$ or $D$ behavior from the agent that received the highest payoff in its neighborhood of $n=5$. The parameters of the payoff matrix, eq. (雨) have been choosen in region $\mathbf{A}$ of the phase diagram, Fig. 6, where we found the spatial coexistence defectors (as the minority) and cooperators (as the majority), the payoff values $a_{\theta}^{s}$ are given by eq. (33).

For the computer simulations, we have again used the initial spatial configurations shown in Fig. 7, i.e. either one small cluster of cooperators, or a random intial distribution of cooperators and defectors with different intial frequencies. Fig. 19 - that shall be compared to Fig. 8 - shows that in the modified case the domain of cooperators considerably increases, i.e. it reaches $f=0.95$ compared to $f=0.52$. Further the dynamics occurs faster (compare the snapshot after 30 generations in Fig. 8 with the one after 20 generations in Fig. 19) and the border between the two domains remains planar during the evolution.
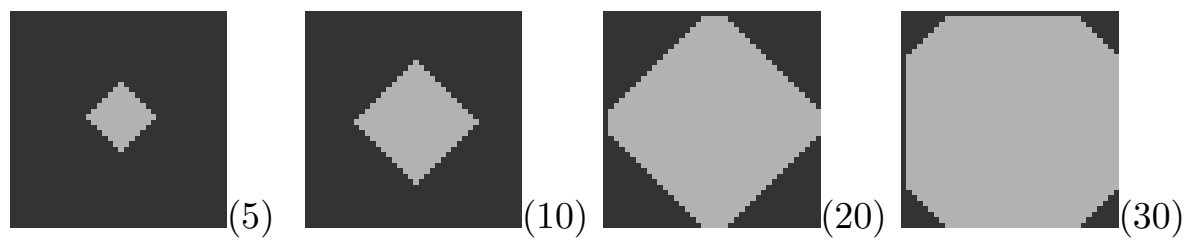

Figure 19: Time series of the spatial distribution of cooperators (grey) and defectors (black) for the modified game, eq. (50). The time is given by the numbers of generations in brackets. Initial condition: One cluster of 9 cooperators, Fig. If(left). Parameters for the payoff matrix, eq. (4): $\{R ; S ; T ; P\}=\{3.0 ; 0.0 ; 3.5 ; 0.5\}$ (region $\mathrm{A}$ ).
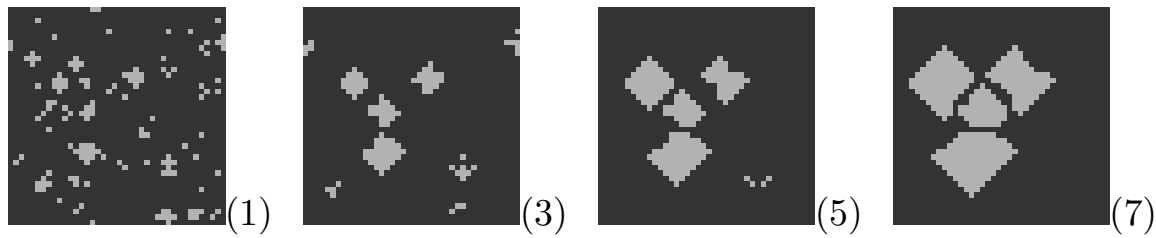

Figure 20: Time series of the spatial distribution of cooperators (grey) and defectors (black) for the modified game, eq. (50). The time is given by the numbers of generations in brackets. Initial condition: $f(0)=0.5$, random spatial distribution of cooperators and defectors, Fig. 7 (right). Parameters: $\{R ; S ; T ; P\}=\{3.0 ; 0.0 ; 3.5 ; 0.5\}$ (region A). We note that the further evolution leads to a final spatial distribution similar to the one shown in Fig. 22(left).

The influence of the larger neighborhood on the evolution of the spatial patterns becomes more 
Frank Schweitzer, Laxmidhar Behera, Heinz Mühlenbein:

Evolution of Cooperation in a Spatial Prisoner's Dilemma

Advances in Complex Systems, vol. 5, no. 2-3, pp. 269-299

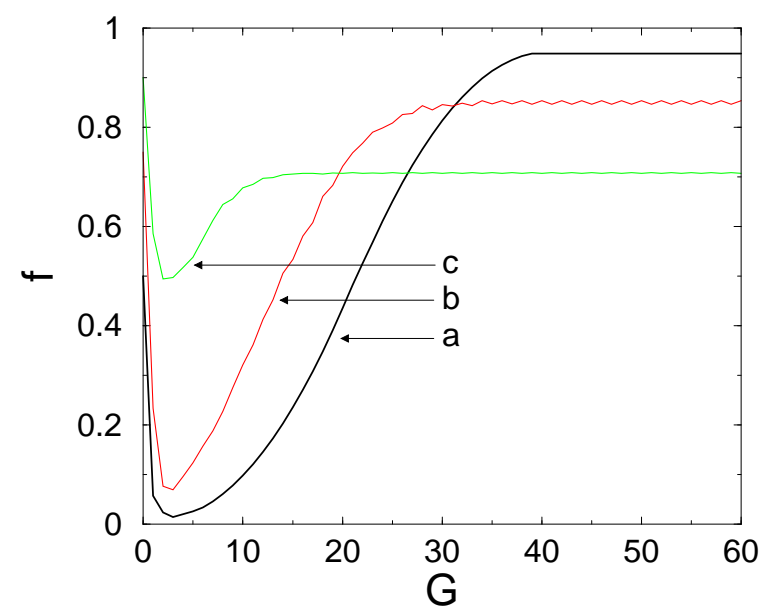

Figure 21: Global frequency of cooperators, $f$, eq. (11), vs. time (number of generations) for the modified game, eq. (50). The data have been obtained from simulations with three different initial frequencies: (a) $f(0)=0.5$, (b) $f(0)=0.75$, (c) $f(0)=0.9$. See also Fig. 22 for the final spatial distributions. Parameters: $\{R ; S ; T ; P\}=\{3.0 ; 0.0 ; 3.5 ; 0.5\}$ (region A).
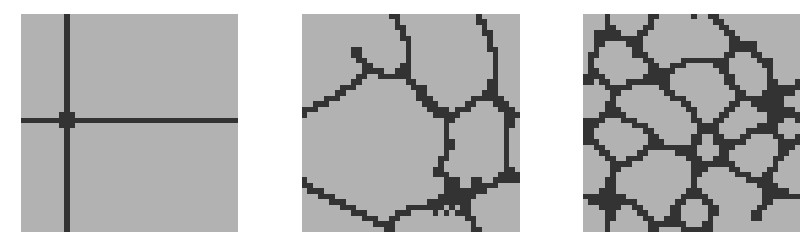

Figure 22: Final (steady state) spatial distribution of cooperators (grey) and defectors (black) for the modified game, eq. (50). Initial condition: random spatial distribution of cooperators and defectors, left: $f(0)=0.5$, middle: $f(0)=0.75$, right: $f(0)=0.9$. Parameters: $\{R ; S ; T ; P\}=$ $\{3.0 ; 0.0 ; 3.5 ; 0.5\}$ (region A).

visible when we start the simulations from a random initial distribution, as shown in Fig. 20. The corresponding evolution of the global frequency $f(G)$ is shown in Fig. 21(a). These figures should be compared to Fig. 2 and Fig. 3. Starting with an initial frequency $f(0)=0.5$, we find that the cooperators during the first three generations almost cease to exist, they survive only in a few rather small clusters. But it is worth to be noticed that this situation then changes drastically: the small clusters grow into a few large domains that are separated by only tiny borders formed by defectors. Thus, compared to the previous simulations, we now find (i) less separated domains, and (ii) a much greater domination of the cooperators in the final state, i.e. $f=0.95$ in this case. Interestingly, this domination becomes the greater, the less the inital frequency is (cf. Fig. 21) but a the same time also the risk increases that cooperators die out during the first generations. If 
Frank Schweitzer, Laxmidhar Behera, Heinz Mühlenbein:

Evolution of Cooperation in a Spatial Prisoner's Dilemma

Advances in Complex Systems, vol. 5, no. 2-3, pp. 269-299

they survive, their "comeback" is overwhelming.

Fig. 22 shows the spatial final distributions that correspond to the different frequencies in Fig. 21. We notice that the different initial frequencies $f(0)$ do not only have a strong impact on final frequencies, but also on the number of domains formed during the evolution. The higher the initial frequency of cooperators, the less is the final frequency of cooperators and the more they are splitted into separated domains. This effect was not so pronounced for the simulations shown in Fig. 10 - thus, we may conclude that the modified game, i.e. the consideration of the spatially heterogeneous interaction in the larger neighborhood may lead to nontrivial diversification in the spatial dynamics, which will be investigated in a forthcoming paper.

\section{Conclusion}

In this paper we have investigated the spatial organization of cooperating and defecting agents distributed on a square lattice. From his interaction with other agents, each agent receives a payoff that can be compared to the payoffs of the other agents. Based on this outcome the agent can in the next generation adopt the more successful behaviour, either to cooperate (C) or to defect (D).

Different from a mean-field approach that assumes a panmictic population where each agent interacts with every other agent, we have considered a spatially restricted case, where each agent interacts only with his neighbors. This results in a spatial 5-person game that has been discussed in two variants: (i) each agent interacts only with his four nearest neighbors, (ii) each agent in the neighborhood of 5 interacts with any other agent in this neighborhood. Such a distinction leads to a different number of 2-person games played simultaneously, but independently by each agent in a given neighborhood, i.e. 4 games in the first case and 10 games in the second case.

The main part of the paper is devoted to the first case. Based on the exact calculation of the payoffs for the possible encounters in a given neighborhood, we have derived analytical expressions (in terms of inequalities) to characterize the different spatial organizations of cooperators and defectors. The results are concluded in a phase diagram for the $\{T, P\}$ parameter space of the possible payoffs for defectors, Fig. 6. We could identify five different dynamical regimes (A-E), each characterized by a distinct spatiotemporal dynamics and a corresponding final spatial distribution. We found that for arbitrary initial conditions parameters choosen from regions E and D will always lead to a spatial invasion of the defectors and eventually results in a complete extinction of the cooperators. This steady state agrees with the steady state of the mean-field dynamics, where the invasion of defectors is the only possible outcome. I.e., if the values of the payoff matrix are choosen from regions D and $\mathrm{E}$, then space plays no longer a role in the determination of the steady state, that is the same regardless of whether the interaction occurs only between nearest neighbors or between all agents in the system. 
Frank Schweitzer, Laxmidhar Behera, Heinz Mühlenbein:

Evolution of Cooperation in a Spatial Prisoner's Dilemma

Advances in Complex Systems, vol. 5, no. 2-3, pp. 269-299

In addition to that we could also identify parameter regions $(\mathrm{A}, \mathrm{B}, \mathrm{C})$ characterized by a spatial coexistence between cooperators and defectors. A detailed analysis could reveal the conditions under which the cooperators could survive either as a majority organized in large spatial domains, or as a minority organized in small non-stationary domains or in small clusters.

The analytical results obtained have been further applied to a spatial game introduced by Nowak and May [32]. The parameter findings obtained there by means of computer simulations could be confirmed by our analytical approach.

In the last part of the paper, we have focussed on the variant (ii) of the 5-person game, where each agent interacts with any other agent in his neighborhood. If this "true" 5-person game is put on a rectangular lattice - as we did here to our knowledge for the first time - it results in interesting effects. Since each agent is part of different spatial 5-person games (played in his immediate neighborhood) the modification eventually leads to the consideration of the secondnearest neighbors. We could show that the interaction in this larger neighborhood can be described as a non-uniform spatial game, where each agent plays 2-person games more frequently with his adjacent neighbors than with his second-nearest neighbors. As the result, we found by means of computer simulations that during the first stages of the evolution the risk considerably increases that the cooperators ceases to exist, but if they survive, they can become a much larger majority than in the "simplified" case (i). In turn, it is the defectors that only survive in thin borders. This is very interesting since true 5-person games are rather complex, and it is known from mean-field investigations that achieving cooperation becomes even more difficult in multi-person games. Thus, the analytical investigations in this paper shall be also applied to the true spatial 5-person game, in order to reveal its critical conditions.

We can conclude that space indeed plays a definite role in the evolution of cooperation, because a spatially restricted interaction may lead to a global cooperation, even if individual rationality tilts toward defection. Even in a one-shot PD game it is possible to find a majority of cooperators, provided a locally restricted interaction is considered.

\section{References}

[1] V. Akimov and M. Soutchanski. Automata simulation of n-person social dilemma games. Journal of Conflict Resolution, 38:138-148, 1994.

[2] Eizo Akiyama and Kunihiko Kaneko. Evolution of cooperation, differentiation, complexity and diversity in an iterated three-person game. Artificial Life, 2:293-304, 1995.

[3] Robert Axelrod. The evolution of Cooperation. Basic Books, New York, 1984.

[4] Robert Axelrod. The Complexity of Cooperation: Agent-Based Models of Competition and Collaboration. University Press, Princeton, N.J., 1999. 
Frank Schweitzer, Laxmidhar Behera, Heinz Mühlenbein:

Evolution of Cooperation in a Spatial Prisoner's Dilemma

Advances in Complex Systems, vol. 5, no. 2-3, pp. 269-299

[5] Robert Axelrod and William Hamilton. The evolution of cooperation. Science, 211:1390-1396, March 271981.

[6] Laxmidhar Behera, Frank Schweitzer, and Heinz Muhlenbein. The invasion of cooperation in heterogeneous populations, i: Thresholds and attractor basins. Journal of Mathematical Sociology, 2002. Submitted.

[7] B. Robert Boyd. Mistakes allow evolutionary stability in the repeated prisoner's dilemma game. J. of Theoretical Biology, 136:47-56, 1989.

[8] Robert Boyd and Jeffrey P. Lorberbaum. No pure strategy is evolutionarily stable in the repeated prisoner's dilemma game. Nature, 327:58-59, May 1987.

[9] Paul Darwen and Xin Yao. Coevolution in iterated prisoner's dilemma with intermediate levels of cooperation: Application to missile defense. Int. J. of Computational Intelligence and Applications, 2(1), March 2002.

[10] M. Doebeli, A. Blarer, and M. Ackermann. Population dynamics, demographic stochasticity, and the evolution of cooperation. Proc. Natl Acad. Sci, USA, 94:5167-5171, 1997.

[11] L. A. Dugatkin. N-person games and the evolution of cooperation: a model based on predator inspection in fish. J. Theoretical Biology, 142:123-135, 1989.

[12] Rick Durrett. Stochastic spatial models. SIAM Review, 41(4):677-718, December 1999.

[13] Joseph Farrell and Roger Ware. Evolutionary stability in the repeated prisoner's dilemma. Theoretical Population Biology, 36:161-166, 1989.

[14] D. B. Fogel. On the relationship between the duration of an encounter and evolution of cooperation in the iterated prisoner's dilemma. Evolutionary Computation, 3(3):349-363, 1995.

[15] N.S. Glance and B. A. Huberman. The outbreak of cooperation. Journal of Mathematical sociology, 17(4):281-302, 1993.

[16] N.S. Glance and B. A. Huberman. The dynamics of social dilemmas. Scientific American, 270:76-81, 1994.

[17] P. Grim. The undecidability of the spatialized prisoner's dilemma. Theory and Decision, 42 (1):53-80, 1997.

[18] Garrett Hardin. The tragedy of the commons. Science, 162:1243-1248, 1968.

[19] Robert Hoffmann. Twenty years on: The evolution of cooperation revisited. Journal of Artificial Societies and Social Simulation, 3(2), 2000. 
Frank Schweitzer, Laxmidhar Behera, Heinz Mühlenbein:

Evolution of Cooperation in a Spatial Prisoner's Dilemma

Advances in Complex Systems, vol. 5, no. 2-3, pp. 269-299

[20] Oliver Kirchkamp. Spatial evolution of automata in the prisoner's dilemma. Journal of economic behavior and organization, 43:239-262, 2000.

[21] David Kraines and Vivian Kraines. Learning to cooperate with Pavlov: an adaptive strategy for the iterated prisoner's dilemma with noise. Theory and Decision, 35:107-150, 1993.

[22] Steven L. Lima. Iterated prisoner's dilemma: An approach to evolutionary stable cooperation. American Naturalist, 134(5):828-834, Nov 1989.

[23] K. Lindgren and J. Johansson. Coevolution of strategies in n-person prisoner's dilemma. In Towards a comprehensive dynamics of evolution. Santa Fe Institute, October 1998.

[24] K. Lindgren and M. G. Nordahl. Evolutionary dynamics of spatial games. Physica D, 75: 292-309, 1994.

[25] K. Lindgren and N.G. Nordahl. Cooperation and community structure in artificial ecosystems. Artificial Life, 1:15-37, 1994.

[26] Jeffrey Lorberbaum. No strategy is evolutionarily stable in the repeated prisoner's dilemma. Journal of Theoretical Biology, 168:117-130, 1994.

[27] M. Matsushima and T. Ikegami. Evolution of strategies in the three person iterated prisoner's dilemma game. J. of Theoretical Biology, 195:53-67, 1998.

[28] Macy Michael. Natural selection and social learning in prisoner's dilemma. Sociological Methods and Research, 25(1):103-138, 1996.

[29] H. Mühlenbein and R. Höns. Stochastic analysis of cellular automata with applications to the voter model. Advances in Complex Systems, 5(2), 2002. (submitted).

[30] Heinz Mühlenbein. Darwin's continent cycle theory and its simulation by the prisoner's dilemma. Complex Systems, 5:459-478, 1991.

[31] M. Nakamaru, H. Matsuda, and Y. Iwasa. The evolution of cooperation in a lattice-structured population. J. Theor. Biology, 184:65-81, 1997.

[32] M. A. Nowak and Robert M. May. The spatial dilemmas of evolution. International Journal of Bifurcation and Chaos, 3(1):35-78, 1993.

[33] Martin A. Nowak and Robert M. May. Evolutionary games and spatial chaos. Nature, 359: 826-829, October 1992.

[34] Martin A. Nowak and Karl Sigmund. The alternating prisoner's dilemma. Journal of Theoretical Biology, 168:219-226, 1994. 
Frank Schweitzer, Laxmidhar Behera, Heinz Mühlenbein:

Evolution of Cooperation in a Spatial Prisoner's Dilemma

Advances in Complex Systems, vol. 5, no. 2-3, pp. 269-299

[35] M. Oliphant. Evolving cooperation in the non-iterated prisoner's dilemma: The importance of spatial organization. In R. Brooks and Maes, editors, Proceedings Proceedings of the Fourth Artificial Life Workshop, pages 349-352, Boston,MA, 1998.

[36] M. Olson. The logic of collective action: public goods and the theory of groups. Harvard University Press, Cambridge,MA, 1965.

[37] A. Rapoport and A. Chammah. Prisoner's dilemma. University of Michigan Press, Ann Arbor, 1965.

[38] Anatol Rapoport. Prisoner's dilemma: reflections and recollections. Simulation and Gaming, 26(4):489-503, 1996.

[39] F. Schweitzer, L. Behera, and H. Mühlenbein. Frequency dependent invasion in a spatial environment. Physical Review E, 2002. (submitted).

[40] Frank Schweitzer, editor. Modeling Complexity in Economic and Social Systems. World Scientific, Singapore, 2002.

[41] Frank Schweitzer, Jörg Zimmermann, and Heinz Mühlenbein. Coordination of decisions in a spatial agent model. Physica A, 303(1-2):189-216, 2002.

[42] J. Maynard Smith. Evolution and the theory of games. Cambridge University Press, UK, 1982.

[43] G. Szabo and C Toke. Evolutionary prisoner's dilemma game on a square lattice. Physical Review E, 58(1):69-73, July 1998.

[44] Robert Trivers. The evolution of reciprocal altruism. Quarterly Review of Biology, 46:35-57, 1972.

[45] M. H. Vainstein and J. J. Arenzon. Disordered environments in spatial games. Physical Review E, 2002.

[46] Xin Yao and J. Darwen, Paul. An experimental study of n-person iterated prisoner' dilemma games. Informatica, 18:435-450, 1994. vorh. 